\title{
Uncertainty in Retrieving Raindrop Size Distribution from Polarimetric Radar Measurements
}

\author{
HAO HUANG \\ Key Laboratory for Mesoscale Severe Weather, Ministry of Education, and School of Atmospheric Sciences, Nanjing University, \\ Nanjing, and State Key Laboratory of Severe Weather, and Joint Center for Atmospheric Radar Research, \\ Centre of Modern Analysis, Nanjing University, Beijing, China

\section{GUIFU ZHANG} \\ School of Atmospheric Sciences, Nanjing University, Nanjing, China, and School of Meteorology, \\ University of Oklahoma, Norman, Oklahoma \\ Kun Zhao, Su Liu, Long Wen, GANG Chen, AND Zhengwei YANG \\ Key Laboratory for Mesoscale Severe Weather, Ministry of Education, and School of Atmospheric Sciences, Nanjing University, \\ Nanjing, and State Key Laboratory of Severe Weather, and Joint Center for Atmospheric Radar Research, \\ Centre of Modern Analysis, Nanjing University, Beijing, China
}

(Manuscript received 27 June 2018, in final form 25 December 2018)

\begin{abstract}
Drop size distribution (DSD) is a fundamental parameter in rain microphysics. Retrieving DSDs from polarimetric radar measurements extends the capabilities of rain microphysics research and quantitative precipitation estimation. In this study, issues in rain DSD retrieval were studied with simulated and measured data. It was found that a three-parameter gamma distribution model was not suitable for directly retrieving DSD from polarimetric radar measurements. A statistical constraint, such as the shape-slope relation used in the constrained-gamma $(\mathrm{C}-\mathrm{G})$ distribution model, helped to reduce the uncertainties and errors in the retrieval. The inclusion of specific differential phase $\left(K_{\mathrm{DP}}\right)$ measurements resulted in more accurate DSD retrieval and rain physical parameter estimation if the measurement errors were properly characterized in the error minimization analysis (EMA), which was verified using two real precipitation events. The study demonstrated the potential of using full polarimetric radar measurements to improve rain DSD retrieval.
\end{abstract}

\section{Introduction}

Drop size distribution (DSD) is a fundamental property of rain microphysics. Rain DSD, denoted as $N(D)$, is defined as the numbers of raindrops in a unit volume per unit size bin (with unit $\mathrm{mm}^{-1} \mathrm{~m}^{-3}$ ). The sizes of raindrops are represented using the equivolume diameter $D(\mathrm{~mm})$. Different moments of $N(D)$ are used alone or combined to calculate physical and statistical parameters of rain, including rainfall rate $R\left(\mathrm{~mm} \mathrm{~h}^{-1}\right)$, liquid water content $W\left(\mathrm{~g} \mathrm{~m}^{-3}\right)$, mass-weighted mean diameter $D_{m}(\mathrm{~mm})$, and total number concentration $N_{t}\left(\mathrm{~m}^{-3}\right)$. Other parameters regarding these physical

Corresponding author: Guifu Zhang, guzhang1@ou.edu; Kun Zhao, zhaokun@nju.edu.cn processes, for example, the evaporation rate and the accretion rate, are also related to DSDs (Zhang et al. 2006).

A disdrometer is an in situ instrument used to measure DSDs. A disdrometer usually has a limited sampling area, and the spatial and temporal variabilities of weather systems make it difficult to obtain comprehensive measurements of DSDs (Bringi et al. 2015). Polarimetric radars typically have fine spatiotemporal resolutions and large coverage, which can measure the microphysical properties of raindrops using horizontally and vertically polarized channels. The measurements made by polarimetric radars, namely, horizontal reflectivity factor $Z_{\mathrm{H}}(\mathrm{dBZ})$, differential reflectivity $Z_{\mathrm{DR}}(\mathrm{dB})$, and specific differential phase $K_{\mathrm{DP}}\left({ }^{\circ} \mathrm{km}^{-1}\right)$, are related to the DSDs (Cao et al. 2012). Therefore, it is possible to retrieve an estimated $N(D)$ from the 
polarimetric measurements. Because only three types of measurements $\left(Z_{\mathrm{H}}, Z_{\mathrm{DR}}\right.$, and $\left.K_{\mathrm{DP}}\right)$ are available for the quantitative retrieval, $N(D)$ should be represented using simplified DSD models containing only a few parameters. The gamma distribution $N(D)=N_{0} D^{\mu} \exp (-\Lambda D)$, $D_{\min } \leq D \leq D_{\max }$ is widely used to represent natural rain spectra, where $N_{0}\left(\mathrm{~mm}^{-1} \mathrm{~m}^{-3}\right)$ is the number concentration parameter, $\mu$ (unitless) is the shape parameter, $\Lambda\left(\mathrm{mm}^{-1}\right)$ is the slope parameter, and $D_{\min }(\mathrm{mm})$ and $D_{\max }(\mathrm{mm})$ are the minimum and maximum diameters of raindrops respectively (Ulbrich 1983; Testud et al. 2001).

Because most DSD variabilities can be accounted for by a DSD model with 2 degrees of freedom (Morrison et al. 2019), the gamma distribution was usually further constrained for DSD retrieval. Seliga and Bringi (1976) proposed to calculate $R$ and the median volume diameter $D_{0}(\mathrm{~mm})$ from $Z_{\mathrm{H}}$ and $Z_{\mathrm{DR}}$ based on the exponential distribution, which assumes $\mu=0$ in the gamma distribution. Zhang et al. (2001) used the gamma distribution constrained by a statistical relation between $\mu$ and $\Lambda$ in retrieval, which was cross verified with the relation between $D_{m}$ and the mass spectrum standard deviation $\sigma_{m}$ (mm; Zhang et al. 2003; Moisseev and Chandrasekar 2007). Cao et al. (2008) also found that the $\mu-\Lambda$ relation may vary in different climate regions (Cao and Zhang 2009). With the constraint of the $\mu-\Lambda$ relation, the degrees of freedom in the gamma distribution is reduced to 2 for DSD retrieval. Testud et al. (2001) demonstrated that the average normalized DSD spectra with 2 degrees of freedom were stable. Later on, Lee et al. (2004) proposed to use a double-moment normalization method to express DSD as a combination of two DSD moments and a double-moment normalized function. Recently, this method was also adapted for X band (Raupach and Berne 2017). Williams et al. (2014) proposed the retrieval of DSD on the basis of the normalized gamma distribution constrained by a statistical relation between $D_{m}$ and $\sigma_{m}$. In the Self-Consistent with Optimal Parameterization attenuation correction and Microphysics Estimation (SCOP-ME) algorithm, a statistical relation between $D_{0}$ and $\mu$ was used (Anagnostou et al. 2013; Kalogiros et al. 2013).

Using the DSD models with 2 degrees of freedom, the DSD parameters and $N(D)$ can be explicitly estimated from two measurements of rain; $Z_{\mathrm{H}}$ and $Z_{\mathrm{DR}}$ are usually chosen as the measurements. However, polarimetric radars also measure the differential phase $\Phi_{\mathrm{DP}}$ and its derivative $K_{\mathrm{DP}}$. Because $K_{\mathrm{DP}}$ is approximately proportional to the fourth moment of $N(D)$, it provides useful information about rain (Huang et al. 2017). Gorgucci et al. (2002) attempted to include $K_{\mathrm{DP}}$ for the estimation of $D_{0}, N_{w}$ (the generalized intercept parameter for the normalized gamma distribution), and $\mu$, as well as the shape of the raindrops, which is known as the beta method.
However, the method proved to be unstable due to the errors in $K_{\mathrm{DP}}$ according to Brandes et al. (2004b). As a result, when adapting the method for $\mathrm{X}$ band, only the data with rainfall rates above $10 \mathrm{~mm} \mathrm{~h}^{-1}$ were used in the study of Park et al. (2005). This method was extended to reduce the impact of the measurement errors in $Z_{\mathrm{DR}}$ and $K_{\mathrm{DP}}$ in Bringi et al. (2002). Whether the inclusion of $K_{\mathrm{DP}}$ can improve the retrieval depends on whether useful information is utilized and the effect of measurement errors is reduced at the same time, which has not been comprehensively studied yet. With the inclusion of $K_{\mathrm{DP}}$, there are three available measurements. The most instinctive way is to estimate DSD based on the gamma distribution, which has 3 degrees of freedom. Its feasibility and performance will be studied. Moreover, even when using a DSD model with 2 degrees of freedom (e.g., the gamma distribution constrained by a $\mu-\Lambda$ relation), $K_{\mathrm{DP}}$ can be incorporated using some mathematical methods, for example, the error minimization analysis (EMA), which will be described in later sections. We assessed the impact of $K_{\mathrm{DP}}$ measurements on DSD retrievals based on these models.

The methods used in this study are introduced in section 2. The feasibility and performance of DSD retrieval using the three-parameter gamma distribution (three-parameter GM) are given in section 3. The performance of the retrievals based on the exponential distribution (EXP) and the gamma distribution constrained by a $\mu-\Lambda$ relation [constrained gamma $(\mathrm{C}-\mathrm{G})]$ was used as a reference. A quantitative study of $K_{\mathrm{DP}}$ 's influences on DSD retrievals on the basis of EXP and C-G is presented in section 4. Section 5 presents a comparison of the performance of the DSD retrievals with and without $K_{\mathrm{DP}}$ measurements in a real weather event. The conclusions and further discussion are given in section 6 .

\section{Methods}

According to Cao et al. (2012), the polarimetric variables $\left(Z_{\mathrm{H}}, Z_{\mathrm{DR}}\right.$, and $\left.K_{\mathrm{DP}}\right)$ are related to $N(D)$ via

$$
\begin{aligned}
Z_{\mathrm{H}, \mathrm{V}} & =10 \log _{10}\left[\frac{4 \lambda^{4}}{\pi^{4}\left|K_{w}\right|^{2}} \int_{D_{\min }}^{D_{\max }}\left|S_{\mathrm{h}, \mathrm{v}}(\pi, D)\right|^{2} N(D) d D\right], \\
Z_{\mathrm{DR}} & =Z_{\mathrm{H}}-Z_{\mathrm{V}}, \\
K_{\mathrm{DP}} & =\frac{0.18 \lambda}{\pi} \int_{D_{\text {min }}}^{D_{\text {max }}} \operatorname{Re}\left[s_{\mathrm{h}}(0, D)-s_{\mathrm{v}}(0, D)\right] N(D) d D,
\end{aligned}
$$

where $D(\mathrm{~mm})$ is the equivalent diameters of the raindrops, $\lambda(\mathrm{mm})$ is the radar wavelength, $K_{w}=$ $(\varepsilon-1) /(\varepsilon+2)$ where $\varepsilon$ (unitless) is the relative dielectric 
constant of water, $s_{\mathrm{h}, \mathrm{v}}(\alpha, D)(\mathrm{mm})$ is the complex scattering amplitude at the horizontal or vertical polarization for raindrops of diameter $D$, with the parameter $\alpha$ being the angle between the incident and scattering directions (in radian), and $\operatorname{Re}(\cdot)$ means the real part of a complex number (Doviak and Zrnić 1993; Bringi and Chandrasekar 2001; Zhang et al. 2001; Cao et al. 2012). Note that the reflectivity factors in linear scale are in units of $\mathrm{mm}^{6} \mathrm{~m}^{-3}$, and the $Z_{\mathrm{H}}$ and $Z_{\mathrm{V}}$ are the reflectivity factors in decibels. In this study, the scattering amplitudes were calculated with the T-matrix method (Vivekanandan et al.1991; Mishchenko et al. 1996). The axis ratio relation was assumed to be the same as that from Brandes et al. (2002). Since the effect of raindrop oscillations has been taken into account in their axis ratio, no additional canting angle was considered in the simulation of our study. The environmental temperature was set to $20^{\circ} \mathrm{C}$. The radar wavelength was assumed to be $10.7 \mathrm{~cm}$, which is used by operational S-band radars. The $D_{\min }$ and $D_{\max }$ were usually assumed to be 0 and $8 \mathrm{~mm}$ respectively in the retrieval.

To retrieve rain DSDs from polarimetric radar data, a DSD model of two or three free parameters is needed in Eqs. (1)-(3). For example, if the gamma distribution is used, $N_{0}, \mu$, and $\Lambda$ can be obtained from $Z_{\mathrm{H}}, Z_{\mathrm{DR}}$, and $K_{\mathrm{DP}}$ by solving a system of three nonlinear equations. For real applications, there are various errors, including microphysics (MP; DSD, shape, orientation) modeling error, electromagnetic (EM) scattering modeling error, and radar measurement $\left(Z_{\mathrm{H}}, Z_{\mathrm{DR}}\right.$, and $\left.K_{\mathrm{DP}}\right)$ errors. These errors cause uncertainty and sometimes result in no solution in rain DSD retrievals from polarimetric radar data (PRD). If an additional constraint is introduced for the gamma distribution (e.g., EXP or C-G), the inverse problem becomes more complicated. Thus, a DSD retrieval framework based on the EMA was introduced. The EMA is based on the idea of optimization using forward operators. The parameters in the DSD model are refined step by step according to the difference of the radar parameters synthesized from the DSD against the measurements. When the difference is minimized, the DSD is optimally estimated. The measurement errors are taken into account in the EMA by normalizing the terms in the cost functions by the corresponding error terms $\left(\sigma_{Z_{\mathrm{H}}}, \sigma_{Z_{\mathrm{DR}}}\right.$, and $\left.\sigma_{K_{\mathrm{DP}}}\right)$. For EXP and C-G, the terms regarding $K_{\mathrm{DP}}$ can be removed from the optimization by increasing the corresponding error term $\sigma_{K_{\mathrm{DP}}}$ to infinity. Then, only $Z_{\mathrm{H}}$ and $Z_{\mathrm{DR}}$ are required for the calculation of the DSD parameters. The formulas and details of the framework are described in the appendix. Using this framework, the impact of the exclusion of $K_{\mathrm{DP}}$ from the DSD retrieval was demonstrated by the sensitivity experiments described in sections 3 and 4 .
The DSD data used in this study were collected by a two-dimensional video disdrometer (2DVD) located at Nanjing, China, in the summers of 2014 and 2015. The position of the 2DVD was indicated by the black dot shown in Fig. 1. Before collecting data, the 2DVD was calibrated by measuring metal balls with known size dropped into the orifice every year. The time resolution for the DSD integration was $1 \mathrm{~min}$. Total number of the DSD samples used for the simulations in this study was 21739. More details of the dataset can be found in Wen et al. (2016). With the DSD dataset, the $\mu-\Lambda$ relation for the summer precipitation in this region was derived using a method of sorting and averaging based on two parameters (SATP; Cao et al. 2008), resulting in

$$
\mu=-0.01760 \Lambda^{2}+0.9472 \Lambda-1.687 .
$$

To assess the performance of the DSD retrievals, simulated polarimetric data were calculated from the observed DSDs following Eqs. (1)-(3). The polarimetric measurements were simulated by adding Gaussian random errors into the synthetic polarimetric data. After estimating $N(D)$ from these simulations, the physical parameters $\left(R, W, D_{m}\right.$, and $\left.N_{t}\right)$ were derived following the formulas in Zhang (2016). In calculating $R$, the statistical terminal velocity derived by Brandes et al. (2004a) was used. Because $N_{t}$ data usually have a very large dynamic range, they were shown using the logarithmic scale in this study. The physical parameters calculated from the observed DSDs were treated as the truth for natural rain, and the retrievals were compared with them using four common parameters, namely, the correlation coefficient (CC), the fractional error (FE), the bias (BIAS), and the root-mean-square error (RMSE), which were calculated as follows:

$$
\begin{aligned}
\mathrm{CC} & =\frac{\sum_{i=1}^{N}\left(V_{e, i}-\bar{V}_{e}\right)\left(V_{d, i}-\bar{V}_{d}\right)}{\sqrt{\sum_{i=1}^{N}\left(V_{e, i}-\bar{V}_{e}\right)^{2} \sum_{i=1}^{N}\left(V_{d, i}-\bar{V}_{d}\right)^{2}}}, \\
\mathrm{FE} & =\frac{\frac{1}{N} \sum_{i=1}^{N}\left|V_{e, i}-V_{d, i}\right|}{\bar{V}_{d}}, \\
\mathrm{BIAS} & =\frac{1}{N} \sum_{i=1}^{N}\left(V_{e, i}-V_{d, i}\right), \\
\mathrm{RMSE} & =\left[\frac{1}{N} \sum_{i=1}^{N}\left(V_{e, i}-V_{d, i}\right)^{2}\right]^{1 / 2},
\end{aligned}
$$

where $V_{e}$ is the one of the physical parameters $(R, W$, $D_{m}$, and $N_{t}$ ) estimated from the retrieved DSD, and 


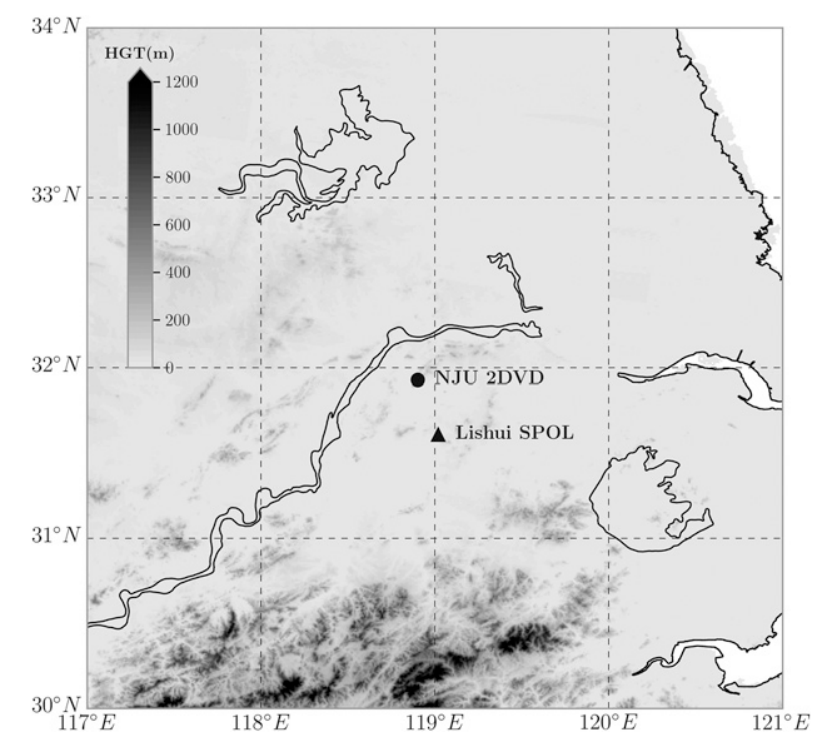

FIG. 1. The topography around Lishui S-Pol (black triangle) and NJU 2DVD (black dot). The elevation of the terrain is indicated as shading.

$V_{d}$ is the truth calculated from the disdrometer data. The overbar indicates the mean value of the $N$ samples.

\section{The feasibility of the gamma distribution-based DSD retrieval}

The gamma distribution has been widely used to characterize rain properties for several decades (Ulbrich 1983). Nevertheless, whether the gamma distribution can be directly used to retrieve the DSD from $Z_{\mathrm{H}}, Z_{\mathrm{DR}}$, and $K_{\mathrm{DP}}$ still remains to be proven. Two major sources of errors in the retrieval are the DSD model error and measurement error, which were analyzed as follows.

\section{a. DSD model error effects}

In the formulation of the DSD retrieval Eqs. (1)-(3), rain DSD is assumed to be gamma distributed so that the three DSD parameters $\left(N_{0}, \mu\right.$, and $\left.\Lambda\right)$ can be solved from the three radar variables $\left(Z_{\mathrm{H}}, Z_{\mathrm{DR}}\right.$, and $\left.K_{\mathrm{DP}}\right)$. In reality, rain DSD may not be exactly gamma distributed. There is a model representation error in the gamma distribution, which will in turn cause an error in DSDs retrieved from polarimetric measurements, which also contain errors. To study the DSD model effects, the EMA-based approach was used with simulated polarimetric radar data. Gamma DSD parameters were estimated from the $Z_{\mathrm{H}}, Z_{\mathrm{DR}}$, and $K_{\mathrm{DP}}$ simulated directly from the $2 \mathrm{DVD}$ data (see section 2). No measurement errors were included in the radar data, and the values of the error terms in the EMA were set to be very small, as explained in the appendix. Then, $R, W, D_{m}$, and $N_{t}$ were calculated from the retrieved DSD for comparison with the corresponding observations (Fig. 2). The results based on the C-G and EXP are provided as references. Overall, the performance of the three-parameter GM was worse than that of the C-G. The deviations of retrieved $R, W$, and $N_{t}$ from the truth were obvious, exhibiting generally larger RMSE, BIAS, and FE values than the C-G. The performance of $D_{m}$ was better than the other three parameters but was still not satisfactory. Although the C-G was based on the gamma distribution, an extra constraint (the $\mu-\Lambda$ relation) made the estimated physical parameters more consistent with those from the observations. The RMSE, BIAS, and FE values were generally low and $C C$ values were high. However, the performance of the EXP was much worse than that of the C-G, even though the EXP was also essentially the gamma distribution with $\mu=0$. The values of RMSE, BIAS, and FE for the EXP were even greater than those for the three-parameter GM, suggesting that simply fixing $\mu=0$ was a nonrealistic constraint. The differences between the physical parameter estimates $(R, W$, $D_{m}$, and $N_{t}$ ) based on the three-parameter GM and the values derived from the DSD observations were mainly caused by its model representation error. Note that, since $R$ and $W$ are close as the DSD moments, they reveal similar behaviors consistently in the comparative study. Thus, most of the results for $R$ were not shown in the rest of the study for simplicity.

In studies of rain properties, the observed DSDs are usually fitted to the three-parameter GM. Here, the untruncated-moment method using the second, fourth, and sixth moments were adopted (Vivekanandan et al. 2004; Zhang 2016). The difference between the fitted DSDs and those retrieved from the radar variables clearly illustrated the errors caused by the different models. Comparisons of the physical parameters calculated from the fitted DSDs and those estimated from the retrieval are shown in Fig. 3. In this simulation, we also assumed that the scattering condition was known. If the natural DSDs exactly followed the assumed three-parameter GM, the retrieved DSDs based on the three-parameter GM would be exactly the same as the fitted ones. Nevertheless, there were significant differences between the physical parameters calculated from the fitted DSDs and those estimated from the retrieved DSDs based on the three-parameter GM, which validated the conclusion obtained from Fig. 2. DSD truncation is an important factor that needs to be considered when representing the natural DSD with the gamma distribution (Vivekanandan et al. 2004). As noted earlier, $D_{\max }$ is not easy to determine from polarimetric measurements, and therefore it is usually given an assumed fixed value (e.g., $8 \mathrm{~mm}$ ) in the 

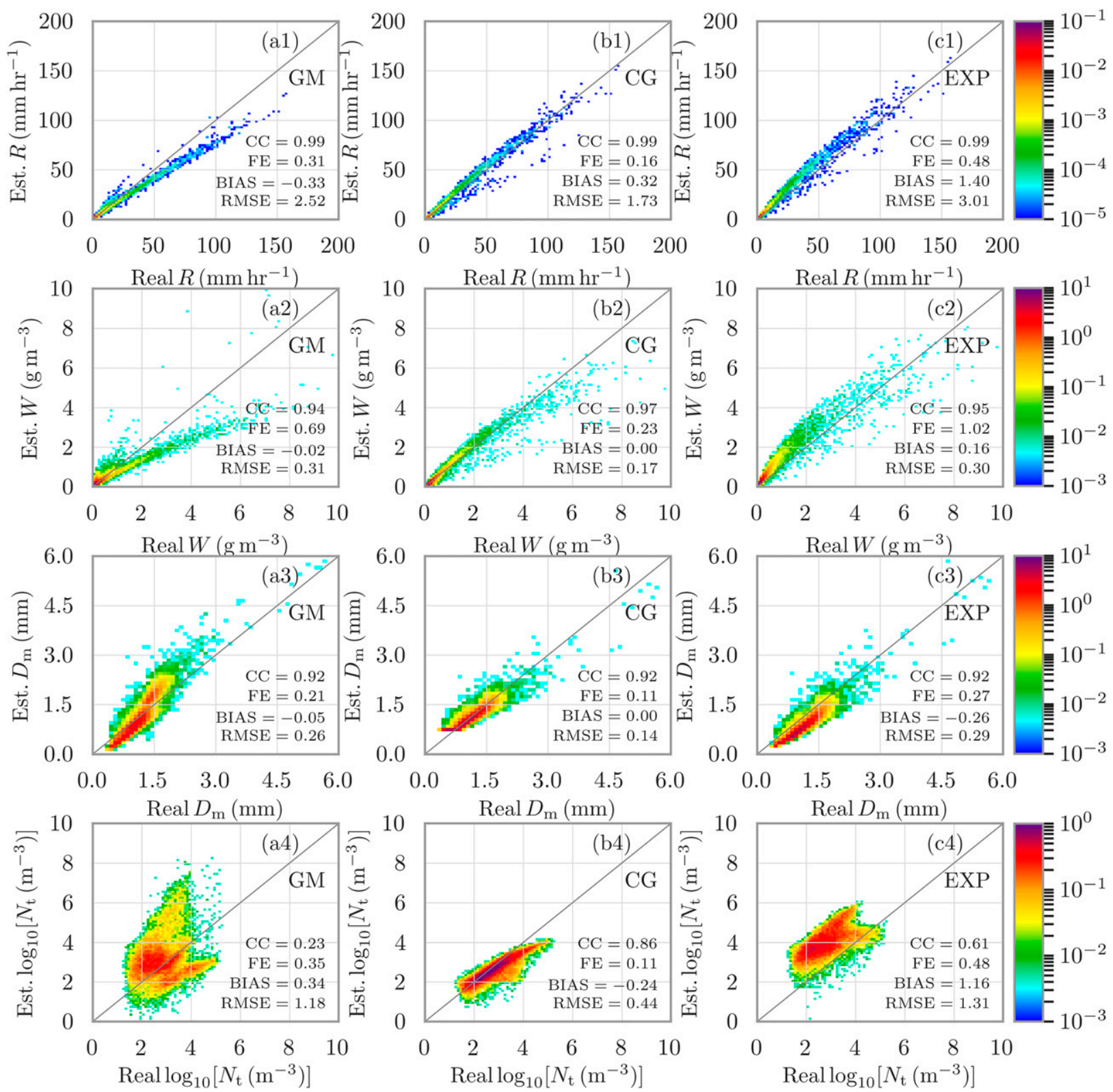

FIG. 2. Comparisons of (a1),(b1),(c1) $R$; (a2),(b2),(c2) $W$; (a3),(b3),(c3) $D_{m}$; and (a4),(b4),(c4) $N_{t}$ calculated from the 2DVD observations against those from the retrieved DSDs. The shading means the two-dimensional probability density. The category sizes of $R, W$, $D_{m}$, and $N_{t}$ are $2 \mathrm{~mm} \mathrm{~h}^{-1}, 0.1 \mathrm{~g} \mathrm{~m}^{-3}, 0.1 \mathrm{~mm}$, and $0.1 \log _{10}\left(\mathrm{~m}^{-3}\right)$, respectively. The DSDs were retrieved from the synthetic $Z_{\mathrm{H}}, Z_{\mathrm{DR}}$, and $K_{\mathrm{DP}}$ based on the three-parameter (a1)-(a4) GM, (b1)-(b4) C-G, and (c1)-(c4) EXP, respectively.

retrieval (Zhang et al. 2001), which is not true for the observed DSD. The inconsistency between the natural DSD and the gamma distribution may also be partially attributed to the microphysical processes of precipitation, for example, the evaporation, collision coalescence, and breakup processes (Kumjian and Ryzhkov 2010; Kumjian and Prat 2014). The size sorting process may cause the "long tail," suggesting an increase in the concentrations of larger raindrops and a decrease in the concentration of small raindrops (Cao and Zhang 2009). Furthermore, the polarimetric variables and the parameters of gamma distribution are not independent, which leads to an ill-posed retrieval problem using the three-parameter GM. As a result, the retrieved physical parameters are not consistent with the truth in Fig. 2.

As indicated by Figs. 2 and 3, the impact of the inconsistency between the natural DSD and the models weakened as extra constraints were used. In this study, 

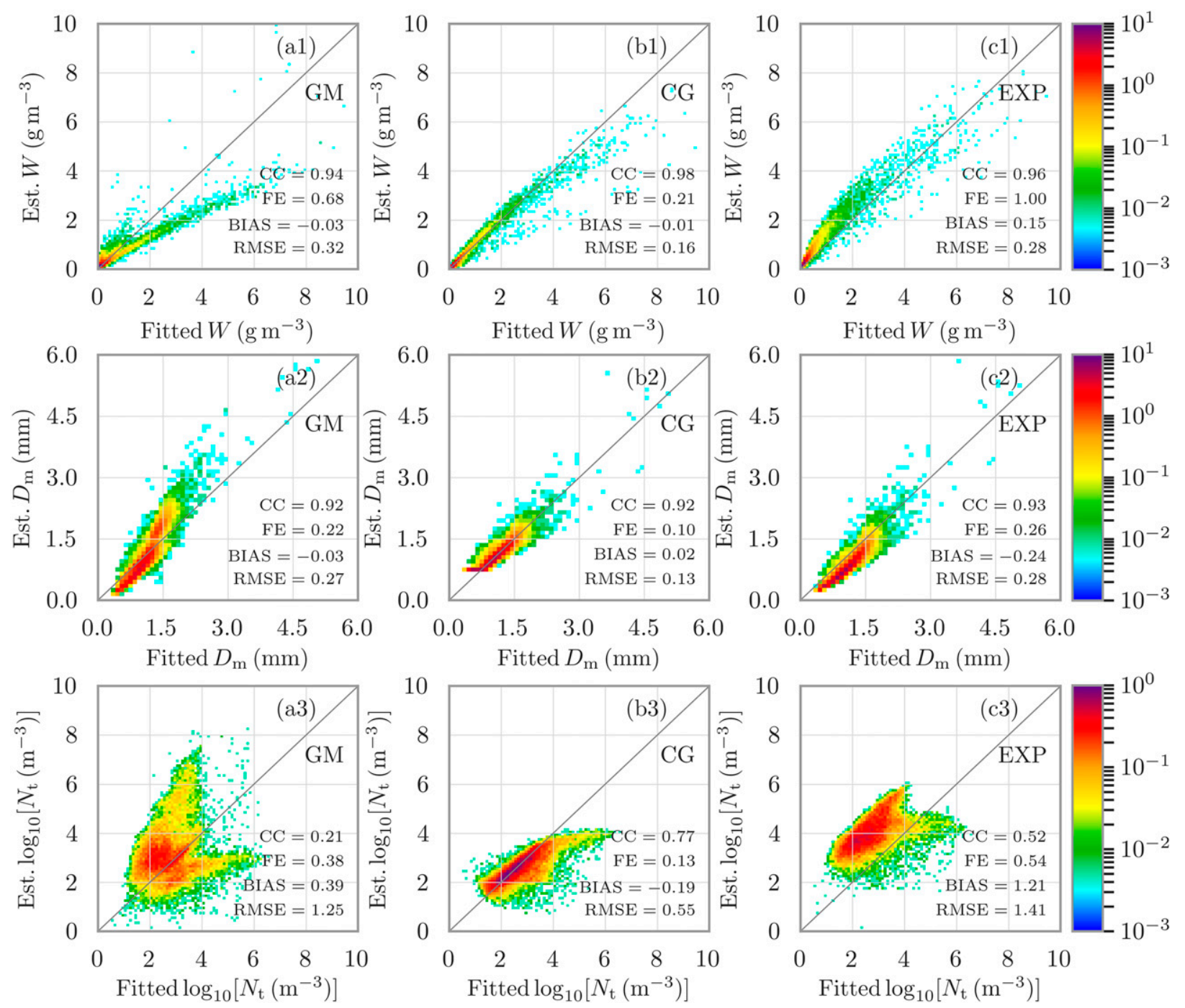

FIG. 3. As in Fig. 2, but for a comparison of the physical parameters calculated from fitted DSDs with those from the retrieved DSDs.

the $\mu-\Lambda$ relation used in $\mathrm{C}-\mathrm{G}$ had better performance than the constraint of EXP $(\mu=0)$. Based on the $\mathrm{C}-\mathrm{G}$, better DSDs and corresponding rain parameters can be retrieved from the synthetic polarimetric variables. The $\mu-\Lambda$ relation can be considered to represent the physical property of natural rain and helps to reduce the uncertainties in DSD retrievals (Zhang et al. 2003).

\section{b. The impacts of simulated measurement errors}

Although polarimetric measurements provide valuable information regarding rain microphysical properties, measurement errors sometimes prevent their effective use and should be rigorously handled in DSD retrievals. To evaluate the impacts of these errors quantitatively, polarimetric measurements were simulated by adding Gaussian random errors to the calculated radar variables from a 2DVD dataset. In this analysis, the standard deviations of the simulated errors for $Z_{\mathrm{H}}$, $Z_{\mathrm{DR}}$, and $K_{\mathrm{DP}}$ were 1 and $0.2 \mathrm{~dB}$ and $0.3^{\circ} \mathrm{km}^{-1}$ respectively (Bringi and Chandrasekar 2001; Ryzhkov et al. 2003; Lee 2006). As such, the error terms in the EMAbased retrieval were assumed to be known, namely $\sigma_{Z_{\mathrm{H}}}=1 \mathrm{~dB}, \sigma_{Z_{\mathrm{DR}}}=0.2 \mathrm{~dB}$, and $\sigma_{K_{\mathrm{DP}}}=0.3^{\circ} \mathrm{km}^{-1}$. The physical parameters of rain calculated from retrieved DSDs based on the three-parameter GM, C-G, and EXP were compared with the truth, as shown in Fig. 4. The physical parameters were less accurate due to the measurement errors. Different DSD models had different levels of sensitivity to the measurement errors. Compared with the results of the C-G, the retrievals based on the three-parameter GM had fewer constraints, making them more sensitive to measurement 

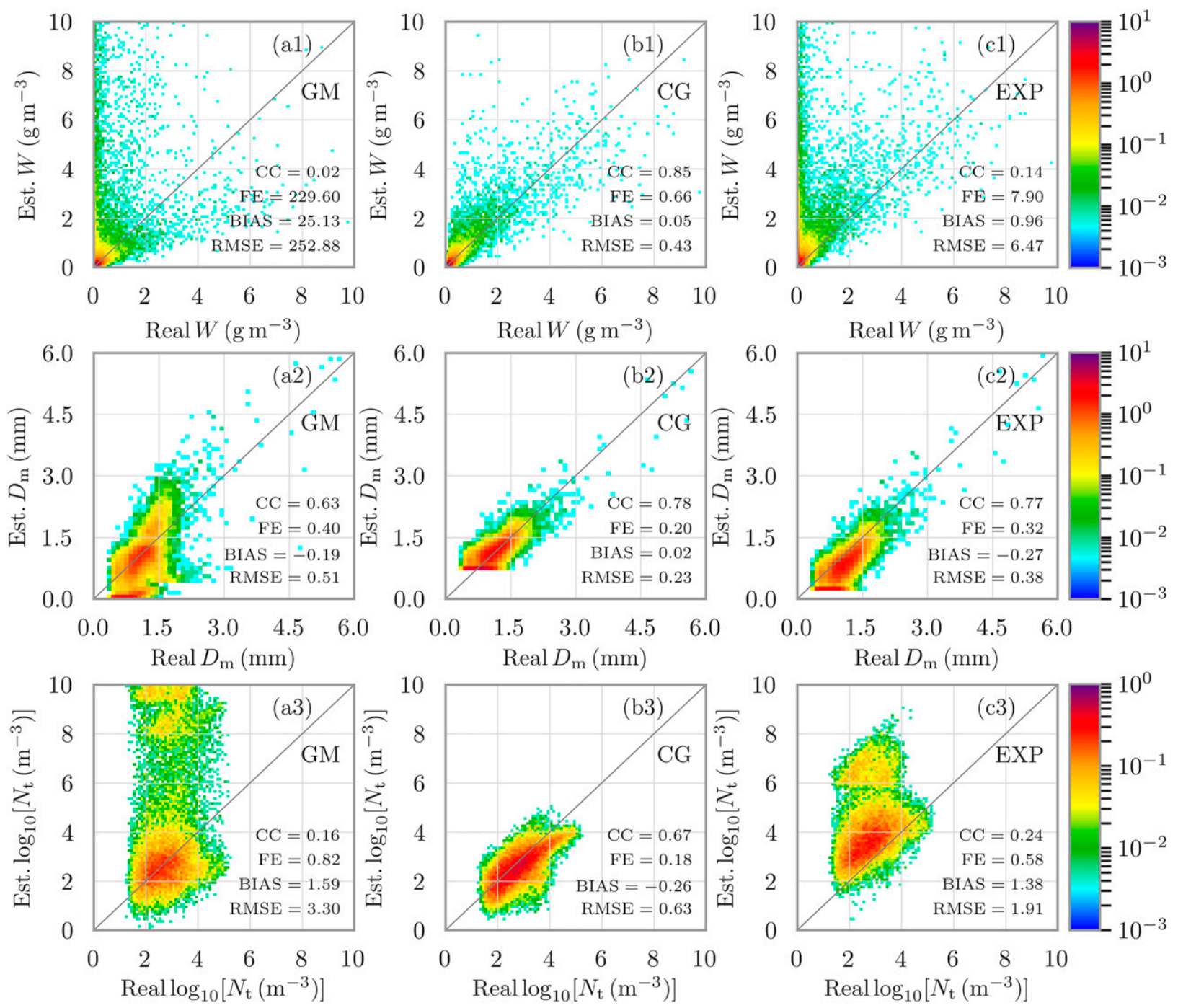

FIG. 4. As in Fig. 2, but with errors in the measurements $\left(\sigma_{Z_{\mathrm{H}}}=1 \mathrm{~dB}, \sigma_{Z_{\mathrm{DR}}}=0.2 \mathrm{~dB}\right.$, and $\left.\sigma_{K_{\mathrm{DP}}}=0.3^{\circ} \mathrm{km}^{-1}\right)$.

errors. Thus, the results based on the three-parameter GM deteriorated significantly, with increased RMSE, $\mathrm{FE}$, and BIAS, and a very low $\mathrm{CC}$. The results of the $\mathrm{C}-\mathrm{G}$ also indicated reduced accuracy in the physical parameters, although the overall performance was satisfactory. The impacts of the measurement errors were diminished by the constraint in the C-G. For the EXP, negative errors in $Z_{\mathrm{DR}}$ and $K_{\mathrm{DP}}$ also affected the estimates of the physical parameters, with greater errors than C-G. The $R, W$, and $N_{t}$ estimates were positively biased, while $D_{m}$ estimates were negatively biased.

It should be noted that no valid estimates can be obtained if $Z_{\mathrm{DR}}$ or $K_{\mathrm{DP}}$ values were negative in conventional retrieval using C-G or EXP (Seliga and Bringi 1976; Zhang et al. 2001). However, the EMA-based approach made it possible to obtain valid estimates under such conditions, which is the advantage of this kind of optimization-based retrieval.

\section{c. The feasibility of the three-parameter GM-based DSD retrieval}

Some conclusions can be drawn from the analyses in the preceding two subsections. Neither radar measurements nor the parameters of gamma distribution were completely independent for natural rain, which makes the retrieval using the three-parameter GM alone ill-conditioned. For natural rain, even without errors in the polarimetric measurements, the retrieved physical parameters based on the threeparameter GM showed significant deviations from the truth. They also showed large sensitivities to the measurement errors. The errors in the radar measurements may increase due to the contaminated 
echoes or echoes with a lower radar signal-to-noise ratio (SNR). Under these circumstances, the results would become even worse than those in the simulations, indicating the importance of additional constraints in the gamma distribution.

\section{The roles of $K_{\mathrm{DP}}$ in DSD retrieval}

\section{a. Comparison of retrievals with and without $K_{\mathrm{DP}}$}

From the quantitative studies in the previous section, we found that the C-G had advantages over the threeparameter GM and EXP when retrieving DSDs from $Z_{\mathrm{H}}, Z_{\mathrm{DR}}$, and $K_{\mathrm{DP}}$ measurements. Before the EMAbased or other optimization-based approaches were developed, DSDs were commonly retrieved from $Z_{\mathrm{H}}$ and $Z_{\mathrm{DR}}$ databased on the DSD models with 2 degrees of freedom, for example, C-G and EXP (Zhang et al. 2001; Brandes et al. 2004a). The $K_{\mathrm{DP}}$ is not a direct radar measurement but is obtained from the range derivative of $\Phi_{\mathrm{DP}}\left(^{\circ}\right)$ over multiple gates. The measurement errors of $K_{\mathrm{DP}}$ are not only from the random errors in $\Phi_{\mathrm{DP}}$ measurements but also from the average of the physical variabilities of rain (Huang et al. 2017). Thus, the resolution volume of $K_{\mathrm{DP}}$ is different from $Z_{\mathrm{H}}$ and $Z_{\mathrm{DR}}$ (Zhang 2016), and many studies only use $Z_{\mathrm{H}}$ and $Z_{\mathrm{DR}}$ in DSD retrievals (Zhang et al. 2001,2003; Zhang 2016). Even so, $K_{\mathrm{DP}}$ measurements still contain valuable information about rain (Gosset et al. 2010), and proper utilization of $K_{\mathrm{DP}}$ may potentially improve DSD retrievals. In the EMA-based approach, if the error term of $K_{\mathrm{DP}}$ is set to infinity, only $Z_{\mathrm{H}}$ and $Z_{\mathrm{DR}}$ measurements will play roles in the retrievals, which allows for a quantitative evaluation of how $K_{\text {DP }}$ measurements affect the retrievals.

First, the intrinsic representatives of $\mathrm{C}-\mathrm{G}$ and EXP for the natural rain were investigated when $K_{\text {DP }}$ was not included. Similar to the simulation in section 3a, no measurement errors were added to the polarimetric data. The results are shown in Fig. 5. The $K_{\mathrm{DP}}$ values were calculated from the retrieved DSD and were compared with the synthetic $K_{\mathrm{DP}}$ from the 2DVD dataset, which was similar to the estimation of $K_{\mathrm{DP}}$ from $Z_{\mathrm{H}}$ and $Z_{\mathrm{DR}}$ based on their self-consistent relations (Vivekanandan et al. 2003; Huang et al. 2017). The high consistency between $K_{\mathrm{DP}}$ calculated from the retrieved DSD and the 2DVD dataset [Figs. 5a(1),b(1)] indicated that both $\mathrm{C}-\mathrm{G}$ and EXP retained self-consistency among the polarimetric variables. If $K_{\mathrm{DP}}$ was excluded from the retrievals, negligible changes appeared in $W$ and $D_{m}$ according to the comparison between Fig. 5 and Figs. $2 \mathrm{~b}$ and $2 \mathrm{c}$. The results for $N_{t}$ and $R$ (not shown) were also similar and are not shown for simplicity. The similarity suggests that, due to the self-consistency of the polarimetric variables, $K_{\mathrm{DP}}$ provides little independent information if no error exists in the polarimetric data.

However, the measurement error effect cannot be underestimated. It is worth investigating how $K_{\mathrm{DP}}$ contributes to DSD retrieval when errors exist in radar measurements. Similar to the experiment described in section $3 \mathrm{~b}$, another retrieval experiment was conducted in which the $K_{\mathrm{DP}}$ measurements were not included. The evaluation of the physical parameters is shown in Fig. 6 . According to the comparison between Fig. 6 and Figs. $4 \mathrm{~b}$ and $4 \mathrm{c}$, the errors in $D_{m}$ and $N_{t}$ did not significantly change when $K_{\mathrm{DP}}$ measurements were not used in the retrievals. However, the errors in $W$ and $R$ slightly increased for the $\mathrm{C}-\mathrm{G}$ but decreased for the EXP, suggesting different roles of the $K_{\mathrm{DP}}$ measurements (see RMSE, FE, BIAS, and CC).

The integrated effects of $K_{\mathrm{DP}}$ may depend on the DSD model. For EXP, if the radar echo intensity is moderate (e.g., $Z_{\mathrm{H}}$ around $40 \mathrm{~dB} Z$ ), the negative $K_{\mathrm{DP}}$ caused by the measurement error may have two different effects, as shown in Table 1 . With no $Z_{\mathrm{DR}}$ errors ( $0 \mathrm{~dB}$ in Table 1$)$, the negative $K_{\mathrm{DP}}$ tended to result in a lower $D_{m}$ and larger $R / W$. However, with a large negative error in the $Z_{\mathrm{DR}}$ measurement (e.g., $-0.55 \mathrm{~dB}$ ), the negative $K_{\mathrm{DP}}$ may result in a very large $\Lambda$ and large $N_{0}$ (note $\mu=0$ for EXP), which represents a high concentration of very small raindrops, resulting in extreme positive biases in the estimated $R$ and $W$ and a negative bias in $D_{m}$. In such a case, the retrieval without $K_{\mathrm{DP}}$ measurements was better than that with $K_{\mathrm{DP}}$ measurements included.

For the C-G, the above situation did not cause severe biases because the concentration of the small drops was suppressed by the positive $\mu$ required by the $\mu-\Lambda$ relation, which mitigated the impacts of measurement errors. As shown by the comparison of Figs. 4 and 6, the incorporation of $K_{\mathrm{DP}}$ measurements enhanced the accuracy of the DSD retrieval, and hence the accuracy of the physical parameters $W$ and $R$. When measurement errors exist, less information is provided by $Z_{\mathrm{H}}$ and $Z_{\mathrm{DR}}$. Under such condition, the incorporation of $K_{\mathrm{DP}}$ made a positive contribution due to selfconsistency of polarimetric variables. Because the C-G has two parameters that need to be determined, the measurement errors generally cannot be removed or canceled if only $Z_{\mathrm{H}}$ and $Z_{\mathrm{DR}}$ are used in the DSD retrieval. By using the EMA-based approach, the DSD was optimized under the statistical constraint of the $\mathrm{C}-\mathrm{G}$, with the consideration of errors in $Z_{\mathrm{H}}, Z_{\mathrm{DR}}$, and $K_{\mathrm{DP}}$. The constraint provided by the $\mu-\Lambda$ relation reflects the physical property of rain (Zhang et al. 2003). Thus, the optimized DSDs from $Z_{\mathrm{H}}, Z_{\mathrm{DR}}$, and $K_{\mathrm{DP}}$ 

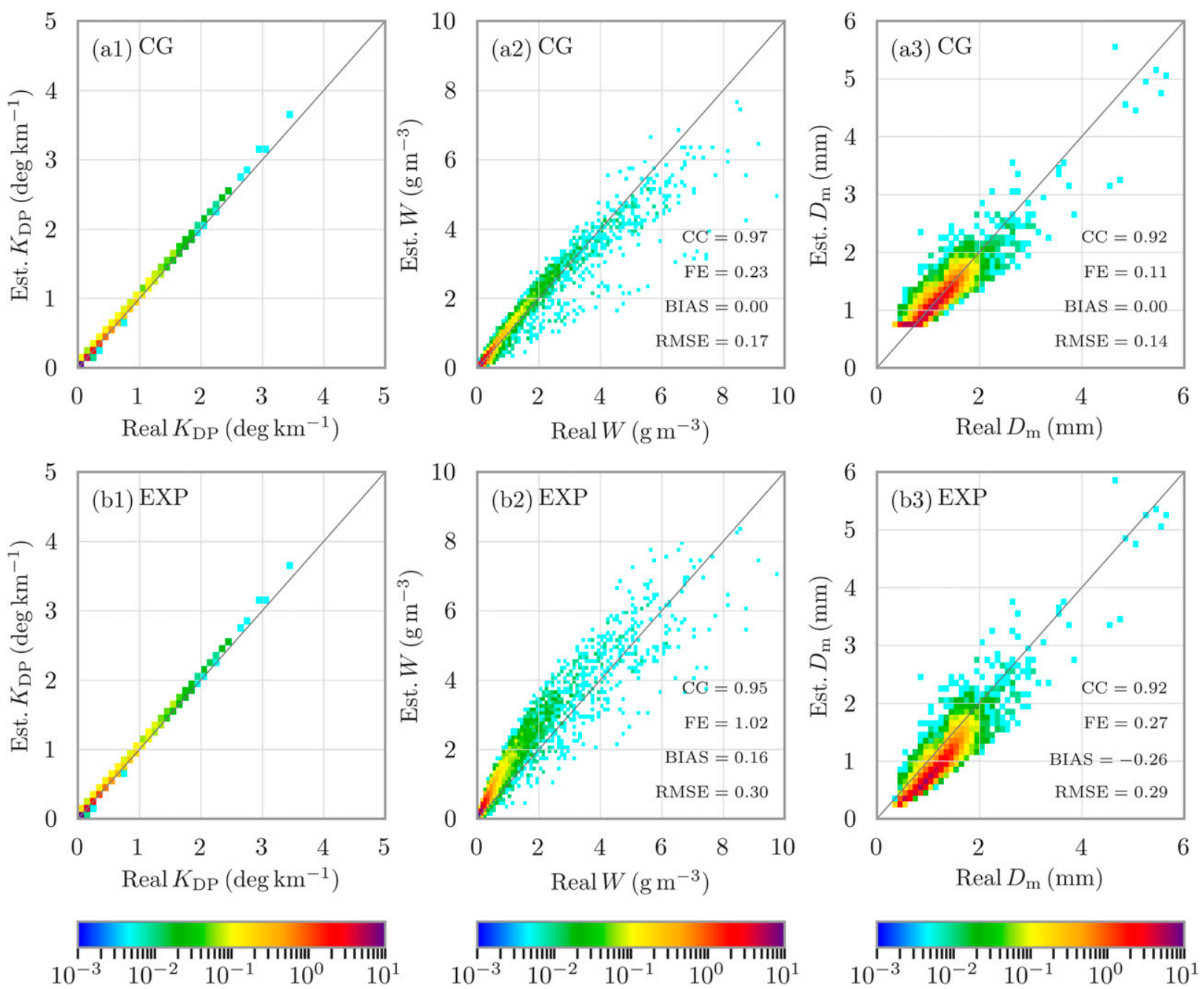

FIG. 5. Comparisons of (a1),(b1) $K_{\mathrm{DP}}$; (a2),(b2) $W$; and (a3),(b3) $D_{m}$ calculated from the retrieved DSDs against those from the 2DVD observations. The shading means the two-dimensional probability density. The category sizes of $K_{\mathrm{DP}}, W$, and $D_{m}$ are $0.1^{\circ} \mathrm{km}^{-1}, 0.1 \mathrm{~g} \mathrm{~m}^{-3}$, and $0.1 \mathrm{~mm}$. The DSDs are retrieved from the synthetic $Z_{\mathrm{H}}$ and $Z_{\mathrm{DR}}$ based on the (a1)-(a3) C-G distribution and (b1)-(b3) EXP, respectively.

were more realistic and less affected by the measurement errors.

\section{b. Quantitative evaluation of the impact of $K_{\mathrm{DP}}$ on the retrieval}

The impact of incorporating $K_{\mathrm{DP}}$ in the retrieval was shown in the previous subsection. The C-G had an advantage over the EXP when we attempted to obtain the most accurate DSD estimates by combining all the useful information of the polarimetric variables. In the earlier experiments, the standard deviation of the $K_{\mathrm{DP}}$ errors was assumed to be equal to $0.3^{\circ} \mathrm{km}^{-1}$. However, in recent years, novel approaches have been proposed for more accurate $K_{\mathrm{DP}}$ estimates, including an approach based on linear programming (Giangrande et al. 2013), the hybrid method (Huang et al. 2017), and the variational approach (Maesaka et al. 2012; Huang et al. 2018). The errors in $K_{\mathrm{DP}}$ can be reduced by incorporating physical constraints or by an optimization using all of the useful information provided by polarimetric radars. On the other hand, when a radar echo is contaminated by nonmeteorological scatterers or when the radar SNR decreases, the backscattering phase or random errors in $\Phi_{\mathrm{DP}}$ can increase significantly. Unfortunately, these can cause larger errors in $K_{\mathrm{DP}}$ (Bringi and Chandrasekar 2001, chapter 6). Moreover, $K_{\mathrm{DP}}$ on a radar gate is calculated based on a series of sequential $\Phi_{\mathrm{DP}}$ data at its neighboring range gates. Large spatial variabilities in these range gates will cause more errors and uncertainties in the $K_{\mathrm{DP}}$ estimates. 

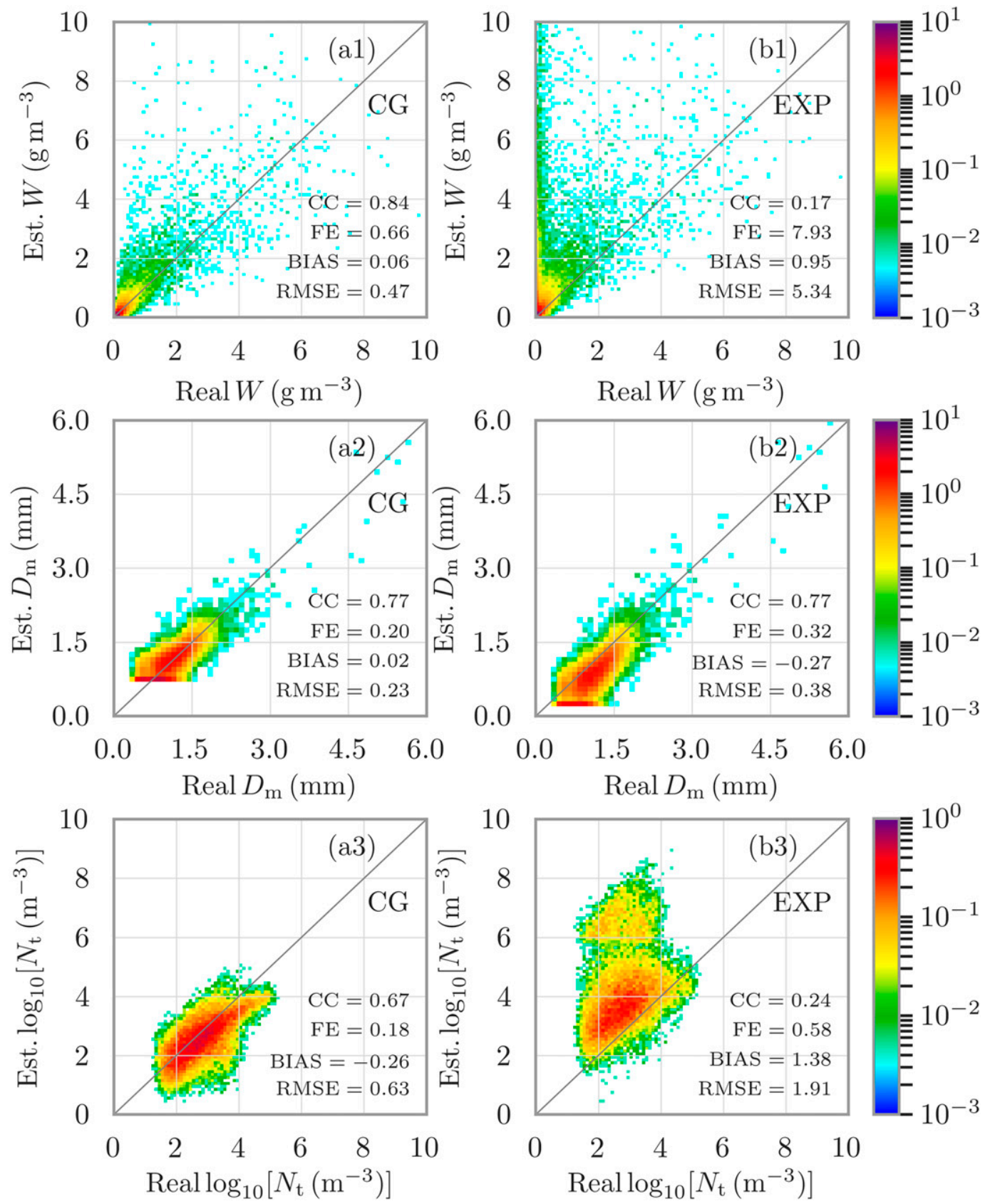

FIG. 6. As in Fig. 4, but for retrievals using $Z_{\mathrm{H}}$ and $Z_{\mathrm{DR}}$ measurements based on the (a1)-(a3) C-G distribution and (b1)-(b3) EXP.

To study quantitatively how $K_{\mathrm{DP}}$ measurements with different error levels can affect the accuracy of DSD retrieval, six groups of comparative experiments were designed. All of these sensitivity experiments used the same group of simulated $Z_{\mathrm{H}}$ and $Z_{\mathrm{DR}}$ measurements with $\sigma_{Z_{\mathrm{H}}}=1 \mathrm{~dB}$ and $\sigma_{Z_{\mathrm{DR}}}=0.2 \mathrm{~dB}$ (see section $3 \mathrm{~b}$ for the simulation of radar measurements). In the control experiment, the $K_{\mathrm{DP}}$ measurements were not used in the retrievals. In the other five groups of sensitivity experiments, we used simulated $K_{\mathrm{DP}}$ measurements with different levels of Gaussian random error and standard deviations ranging from $0.1^{\circ}$ to $0.5^{\circ} \mathrm{km}^{-1}$, with a $0.1^{\circ} \mathrm{km}^{-1}$ interval. DSDs were then retrieved from the simulated radar measurements using the EMA-based approach. The error terms in the EMA were assumed to be known and correctly used in all sensitivity measurements. Only the $\mathrm{C}-\mathrm{G}$ was used as the model for DSD retrieval because the EXP and the 
TABLE 1 . The performance of the retrieved physical parameters $\left(R, W\right.$, and $\left.D_{m}\right)$, with two different levels of errors included. The retrieval was based on the polarimetric data calculated from an observed DSD $\left(Z_{\mathrm{H}}=39.962 \mathrm{dBZ}, Z_{\mathrm{DR}}=0.597 \mathrm{~dB}, K_{\mathrm{DP}}=0.245^{\circ} \mathrm{km}^{-1}\right)$.

\begin{tabular}{|c|c|c|c|c|c|}
\hline & & & $R\left(\mathrm{~mm} \mathrm{~h}^{-1}\right)$ & $W\left(\mathrm{~g} \mathrm{~m}^{-3}\right)$ & $\overline{D_{m}(\mathrm{~mm})}$ \\
\hline & & Truth & 24.01 & 1.43 & 1.3 \\
\hline$\varepsilon_{Z_{\mathrm{H}}}=0.0$ & With $K_{\mathrm{DP}}$ & C-G & 24.82 & 1.46 & 1.31 \\
\hline$\varepsilon_{Z_{\mathrm{DR}}}=0.0$ & & EXP & 30.45 & 2.13 & 1.07 \\
\hline \multirow[t]{2}{*}{$\varepsilon_{K_{\mathrm{DP}}}=-0.6$} & Without $K_{\mathrm{DP}}$ & C-G & 26.19 & 1.55 & 1.3 \\
\hline & & EXP & 32.21 & 2.27 & 1.06 \\
\hline$\varepsilon_{Z_{\mathrm{H}}}=0.0$ & With $K_{\mathrm{DP}}$ & C-G & 118.25 & 11.25 & 0.71 \\
\hline$\varepsilon_{Z_{\mathrm{DR}}}=-0.55$ & & EXP & 1040.75 & 344.95 & 0.2 \\
\hline \multirow[t]{2}{*}{$\varepsilon_{K_{\mathrm{DP}}}=-0.6$} & Without $K_{\mathrm{DP}}$ & C-G & 124.22 & 11.81 & 0.71 \\
\hline & & EXP & 281.2 & 47.74 & 0.39 \\
\hline
\end{tabular}

three-parameter GM exhibit clear shortcomings in the DSD retrieval due to the lack of physical constraints.

The dependence of the retrieval accuracy on $K_{\mathrm{DP}}$ errors was then demonstrated with respect to the echo intensity $\left(Z_{\mathrm{H}}\right)$. As shown in Fig. 7, the synthetic $Z_{\mathrm{H}}$ from the DSD was separated into nine bins ranging from 12 to $48 \mathrm{~dB} Z$ with $4-\mathrm{dB}$ intervals, and the CC, BIAS, FE, and RMSE of the physical parameters were calculated within each $Z_{\mathrm{H}}$ bin. The results demonstrated that there was no significant change for $D_{m}$ when the measurement errors in the $K_{\mathrm{DP}}$ changed from $0.1^{\circ}$ to $0.5^{\circ} \mathrm{km}^{-1}$ or even when $K_{\mathrm{DP}}$ measurements were not used in the retrieval. For $W, N_{t}$, and $R$, the accuracy of the retrieval changed when the errors in the $K_{\mathrm{DP}}$ measurements changed. This effect was not significant when the echo intensity $Z_{\mathrm{H}}<35 \mathrm{dBZ}$, while the advantage of using more accurate $K_{\mathrm{DP}}$ measurements was clear when $Z_{\mathrm{H}}>35 \mathrm{~dB} Z$. Note that the threshold $35 \mathrm{dBZ}$ was also adopted by Bringi et al. (2002) to determine whether $K_{\mathrm{DP}}$ should be used for DSD retrieval. The CC values increased and the RMSE, FE, and BIAS decreased when the errors in the $K_{\mathrm{DP}}$ measurements changed, indicating more accurate physical parameters and hence a more accurate DSD. This effect intensified as $Z_{\mathrm{H}}$ increased. Even when including $K_{\mathrm{DP}}$ measurements with relatively large errors $\left(\sigma_{K_{\mathrm{DP}}}=0.5^{\circ} \mathrm{km}^{-1}\right)$, the $W, N_{t}$, and $R$ estimates were still more accurate than those estimated from the retrieval using only $Z_{\mathrm{H}}$ and $Z_{\mathrm{DR}}$ measurements. Note that $N_{t}$ was in logarithmic scale. Thus, only the increase in $\mathrm{CC}$ was significant.

To investigate the underlying reason for this, the synthetic polarimetric variables calculated from the retrieved DSDs were compared with those calculated from the observed DSD data. Only the results for $\sigma_{K_{\mathrm{DP}}}=0.1^{\circ}$ and $0.3^{\circ} \mathrm{km}^{-1}$ as well as those without $K_{\mathrm{DP}}$ are shown in Fig. 8. The comparisons between the estimated and the synthetic polarimetric variables indicate how much error was removed and how much propagated to the estimates. When the DSD was retrieved from two measurements $\left(Z_{\mathrm{H}}\right.$ and $\left.Z_{\mathrm{DR}}\right)$, most errors except the negative $Z_{\mathrm{DR}}$ could not be removed because there was no additional information. As a consequence, the errors in the estimated $K_{\mathrm{DP}}$ shown in Fig. 8a(3) were relatively large compared with those in Figs. $8 \mathrm{~b}(3)$ and $8 \mathrm{c}(3)$. When $K_{\mathrm{DP}}$ was used, the errors in the three measurements could be partially canceled through the optimization because of the additional constraint in C-G. In the EMA-based retrieval, the error terms in the cost function acted as weightings or as a quality index for the measurements. When the errors in the $K_{\mathrm{DP}}$ measurements were reduced, $K_{\mathrm{DP}}$ contributed more to the estimation, especially when the echo intensity was severe (relatively large $Z_{\mathrm{H}}$; see Fig. 7 and Fig. 8). Because $Z_{\mathrm{H}}$ was recorded using the decibel scale, the errors in $Z_{\mathrm{H}}$ had a large impact on the estimation of $W, N_{t}$, and $R$ when $Z_{\mathrm{H}}$ was relatively large. Under such conditions, more useful information in $K_{\mathrm{DP}}$ contributed to the estimates since the effect of the errors in $K_{\mathrm{DP}}$ is linear. When $\sigma_{K_{\mathrm{DP}}}$ decreased, more accurate information in $K_{\text {DP }}$ resulted in more accurate estimation of $W, N_{t}$, and $R$ (Figs. 7a and $7 \mathrm{c}$ ). In the meantime, fewer $Z_{\mathrm{H}}$ errors propagated to the estimates, and $Z_{\mathrm{H}}$ estimates would become more accurate when $Z_{\mathrm{H}}>35 \mathrm{~dB} Z$ [Figs. 8a(1)-c(1)]. However, $Z_{\mathrm{DR}}$ estimates barely changed even when very accurate $K_{\mathrm{DP}}$ measurements were used in the retrieval [Figs. $8 \mathrm{a}(2)-\mathrm{c}(2)$ ], corresponding to quasi invariability in the performance of the $D_{m}$ estimates [Figs. 7b(1)-b(4)].

\section{c. Effects of the inaccurate characterization of the $K_{\mathrm{DP}}$ error properties}

As stated earlier, the advantage of the EMA-based retrievals was that all the available measurements were used according to their reliabilities, as determined by their error properties (error terms). Thus, accurate retrievals require relatively accurate characterization of the measurement errors. However, the $K_{\mathrm{DP}}$ errors are not easy to estimate quantitatively because they are derived not only from the errors in $\Phi_{\mathrm{DP}}$ measurements but also from the uncertainty in the spatial variabilities 


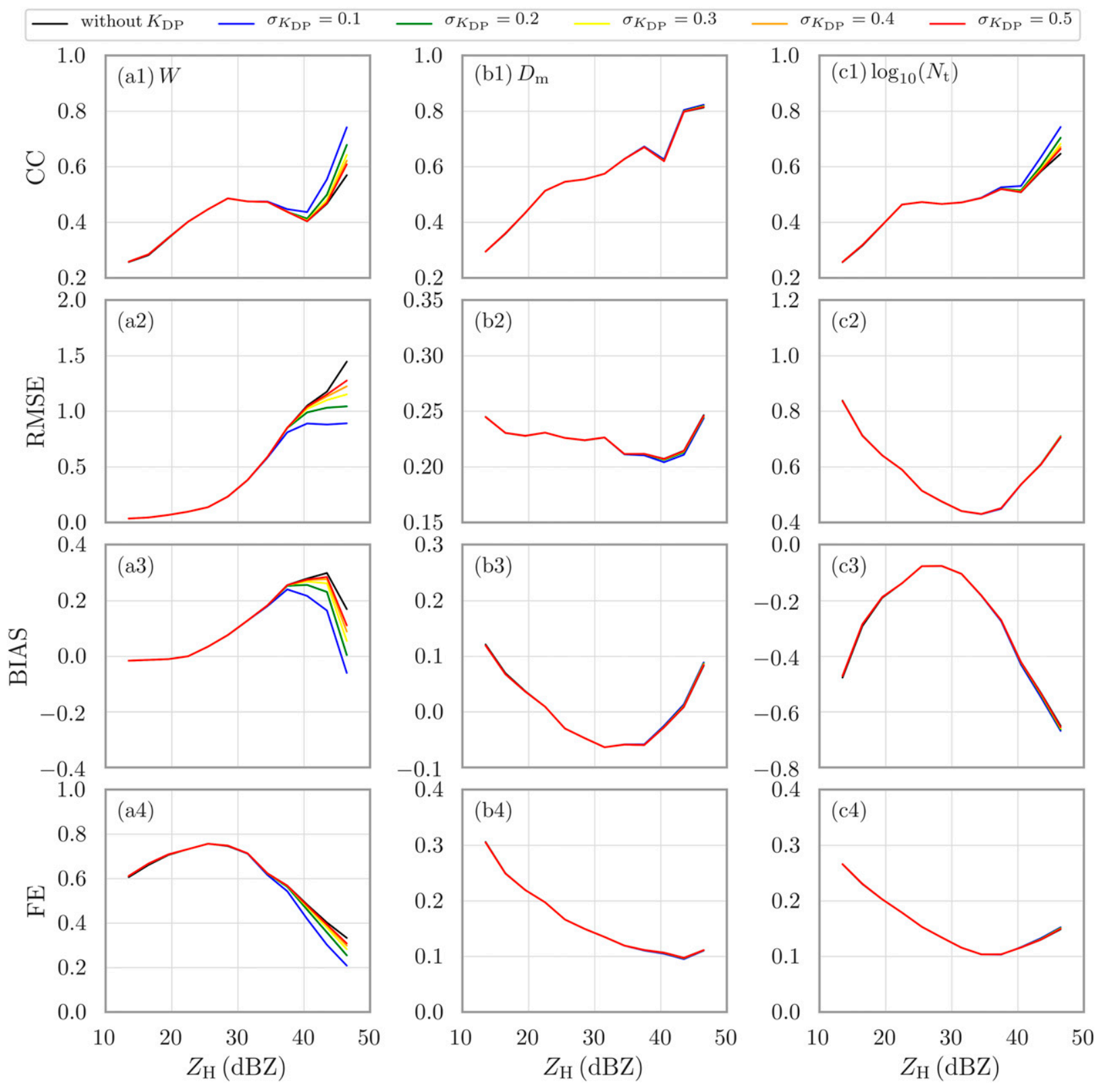

FIG. 7. Comparisons of (a1),(b1),(c1) CC, (a2),(b2),(c2) RMSE, (a3),(b3),(c3) BIAS, and (a4),(b4),(c4) FE corresponding to (a1)-(a4) $W$, (b1)-(b4) $D_{m}$, and (c1)-(c4) $N_{t}$ calculated from the retrieved DSDs without $K_{\mathrm{DP}}$ measurements or with $K_{\mathrm{DP}}$ measurements including different random errors.

of rain properties. We next investigated how the performance of the retrieval changed when inaccurate $K_{\mathrm{DP}}$ error terms were used in the retrieval.

The following experiments were designed based on the earlier ones. Simulated radar measurements were obtained by adding Gaussian simulated errors to the synthetic polarimetric data $\left(\sigma_{Z_{\mathrm{H}}}=1 \mathrm{~dB}, \sigma_{Z_{\mathrm{DR}}}=0.2 \mathrm{~dB}\right.$, $\left.\sigma_{K_{\mathrm{DP}}}=0.3^{\circ} \mathrm{km}^{-1}\right)$. Unlike the previous experiments, the error properties of the $K_{\mathrm{DP}}$ measurements were assumed to be not well known. As listed in Table 2, the error terms $\left(\sigma_{K_{\mathrm{DP}}}\right)$ used in the EMA in the different experiments were $0.1^{\circ}, 0.2^{\circ}, 0.3^{\circ}, 0.4^{\circ}$, and $0.5^{\circ} \mathrm{km}^{-1}$. A retrieval with the accurate $\sigma_{K_{\mathrm{DP}}}\left(0.3^{\circ} \mathrm{km}^{-1}\right)$ was used as a control experiment. From the RMSE, BIAS, FE, and $\mathrm{CC}$ of the physical parameters for each experiment, we found that the physical parameters became less accurate if inaccurate $\sigma_{K_{\mathrm{DP}}}$ values were used. Among the four physical parameters, $D_{m}$ was the least affected. The useful information in the $K_{\mathrm{DP}}$ measurements was insufficiently used if the overestimated $\sigma_{K_{\mathrm{DP}}}$ was used in 

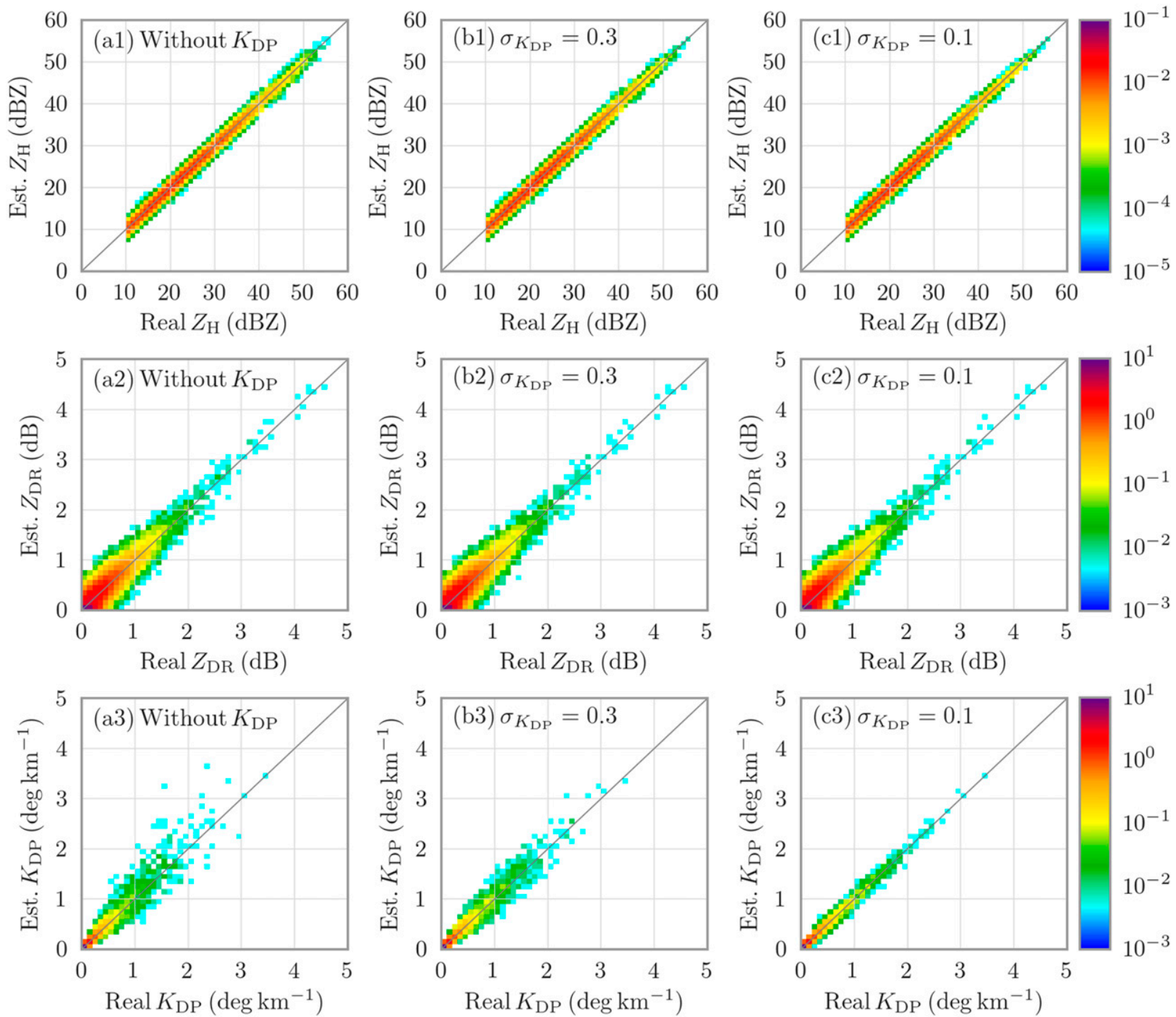

FIG. 8. Comparisons of synthetic (a1),(b1),(c1) $Z_{\mathrm{H}}$, (a2),(b2),(c2) $Z_{\mathrm{DR}}$, and (a3),(b3),(c3) $K_{\mathrm{DP}}$ from the retrieved DSDs against those from the 2DVD data. The shading means the two-dimensional probability density. The category sizes of $Z_{\mathrm{H}}, Z_{\mathrm{DR}}, K_{\mathrm{DP}}, R$, and $D_{m}$ are $1 \mathrm{~dB}, 0.1 \mathrm{~dB}$, and $0.1^{\circ} \mathrm{km}^{-1}$. (a1)-(a3) The results from the retrievals only using $Z_{\mathrm{H}}$ and $Z_{\mathrm{DR}}$ measurements. (b1)-(b3),(c1)-(c3) The results from the retrievals including measurements of different $K_{\mathrm{DP}}$ accuracy $\left(\sigma_{K_{\mathrm{DP}}}=0.3^{\circ} \mathrm{km}^{-1}\right.$ and $\sigma_{K_{\mathrm{DP}}}=0.1^{\circ} \mathrm{km}^{-1}$, respectively).

the EMA. On the other hand, if a $\sigma_{K_{\mathrm{DP}}}$ lower than the real error was used, the measurement errors in $K_{\mathrm{DP}}$ tended to propagate into the DSDs and the corresponding physical parameters. The retrievals under both situations were less accurate than that obtained when using an accurate $\sigma_{K_{\mathrm{DP}}}$ The results from the experiments using overestimated $\sigma_{K_{\mathrm{DP}}}$ values $(0.4$ or 0.5$)$ were generally better than those obtained using underestimated $\sigma_{K_{\mathrm{DP}}}$ values $(0.1$ or 0.2$)$. This is probably because even when the information in $K_{\mathrm{DP}}$ measurements was underutilized, better results than those without $K_{\mathrm{DP}}$ measurements could still be obtained. However, by using underestimated $\sigma_{K_{\mathrm{DP}}}$ values, the $K_{\mathrm{DP}}$ measurements were overly weighted. The useful information in $Z_{\mathrm{H}}$ and
$Z_{\mathrm{DR}}$ measurements was lost and the $K_{\mathrm{DP}}$ errors overly propagated into the retrieval results. For example, even $D_{m}$ can deteriorate when $\sigma_{K_{\mathrm{DP}}}=0.1^{\circ} \mathrm{km}^{-1}$ is used improperly. Therefore, when it is difficult to determine the errors in $K_{\mathrm{DP}}$ measurements, it is better not to use a relatively small $\sigma_{K_{\mathrm{DP}}}$.

\section{DSD retrieval in a real event}

In the above study, simulation experiments based on observed DSD data were used to demonstrate the feasibility of directly retrieving the DSDs from polarimetric measurements based on the three-parameter GM and that including $K_{\mathrm{DP}}$ measurements with accurate error 
TABLE 2. The performance of the estimated physical parameters $\left(R, W, D_{m}\right.$, and $\left.N_{t}\right)$ for rain with different $\sigma_{K_{\mathrm{DP}}}$ used in the error minimization analysis (EMA)-based retrieval.

\begin{tabular}{|c|c|c|c|c|c|c|}
\hline \multicolumn{2}{|c|}{$\sigma_{K_{\mathrm{DP}}}\left({ }^{\circ} \mathrm{km}^{-1}\right)$} & \multirow{2}{*}{$\begin{array}{l}0.1 \\
0.876\end{array}$} & \multirow{2}{*}{$\begin{array}{l}0.2 \\
0.924\end{array}$} & \multirow{2}{*}{$\begin{array}{l}0.3 \\
0.93\end{array}$} & \multirow{2}{*}{$\begin{array}{l}0.4 \\
0.928\end{array}$} & \multirow{2}{*}{$\frac{0.5}{0.927}$} \\
\hline$R\left(\mathrm{~mm} \mathrm{~h}^{-1}\right)$ & $\mathrm{CC}$ & & & & & \\
\hline & $\mathrm{FE}$ & 0.517 & 0.491 & 0.49 & 0.491 & 0.491 \\
\hline & RMSE & 7.655 & 5.643 & 5.423 & 5.511 & 5.616 \\
\hline \multirow{5}{*}{$W\left(\mathrm{~g} \mathrm{~m}^{-3}\right)$} & BIAS & 1.135 & 0.875 & 0.859 & 0.875 & 0.89 \\
\hline & $\mathrm{CC}$ & 0.778 & 0.839 & 0.846 & 0.843 & 0.842 \\
\hline & FE & 0.689 & 0.659 & 0.657 & 0.658 & 0.658 \\
\hline & RMSE & 0.57 & 0.445 & 0.431 & 0.438 & 0.443 \\
\hline & BIAS & 0.07 & 0.051 & 0.05 & 0.051 & 0.052 \\
\hline \multirow[t]{4}{*}{$D_{m}(\mathrm{~mm})$} & $\mathrm{CC}$ & 0.773 & 0.775 & 0.775 & 0.775 & 0.775 \\
\hline & $\mathrm{FE}$ & 0.199 & 0.198 & 0.198 & 0.198 & 0.198 \\
\hline & RMSE & 0.231 & 0.23 & 0.23 & 0.23 & 0.23 \\
\hline & BIAS & 0.019 & 0.02 & 0.02 & 0.02 & 0.02 \\
\hline \multirow{4}{*}{$\log _{10}\left[N_{t}\left(\mathrm{~m}^{-3}\right)\right]$} & $\mathrm{CC}$ & 0.664 & 0.666 & 0.667 & 0.666 & 0.667 \\
\hline & $\mathrm{FE}$ & 0.18 & 0.18 & 0.18 & 0.18 & 0.18 \\
\hline & RMSE & 0.635 & 0.633 & 0.632 & 0.632 & 0.632 \\
\hline & BIAS & -0.253 & -0.255 & -0.255 & -0.255 & -0.255 \\
\hline
\end{tabular}

properties in the EMA-based retrieval led to more accurate DSDs and physical parameter estimates. Their performance will be investigated using real radar observations in the current section.

An S-band polarimetric radar called the Lishui radar (black triangle in Fig. 1) is located about $37 \mathrm{~km}$ to the southeast of the 2DVD. The radar was calibrated with a hollow metal sphere in June 2014, which ensured the accuracy of the radar reflectivity measurements. The $Z_{\mathrm{DR}}$ was calibrated by monitoring the $Z_{\mathrm{DR}}$ variations of the drizzle echo. The echoes with a correlation coefficient $\left(\rho_{\mathrm{hv}}\right)$ less than 0.8 were considered to be potentially contaminated by clutter or other nonmeteorological scatterers and were removed. The adaptive least squares fitting method similar to that adopted by WSR-88D was used to calculate $K_{\mathrm{DP}}$ (Huang et al. 2017); $K_{\mathrm{DP}}$ at each gate was obtained from the least squares fitting of $\Phi_{\mathrm{DP}}$ within a specific fitting window. If the corrected $Z_{\mathrm{H}}$ is above (below) $40 \mathrm{~dB} Z$, the length of the adaptive fitting window was set to 2.5 (9) $\mathrm{km}$. On 12 and 3-4 July 2014, two precipitation systems passed over both the Lishui radar and the 2DVD. DSDs and physical parameters $(R, W$, $D_{m}$, and $N_{t}$ ) were retrieved from the radar measurements. The radar-derived parameters were quantitatively compared with the 2DVD observations to show the performance of different retrieving models.

The precipitation event on 12 July 2014 lasted for about $9 \mathrm{~h}$. Figure 9 shows the plan position indicator (PPI) images of the radar scan at $0.5^{\circ}$ elevation at 0640 UTC. The weather system produced both stratiform and convective precipitation. The $Z_{\mathrm{DR}}$ values were generally lower in the stratiform regions than in the convective regions, with substantial variation in the convective regions, indicating vastly different microphysical properties. The black lines in Fig. 10 show the synthetic polarimetric measurements generated from the 2DVD observations, while the red dots show the polarimetric radar measurements at $0.5^{\circ}$ elevation above the 2DVD site for comparison. The rain DSDs sampled by the 2DVD contained both stratiform and convective precipitation. The vertical distance between the center of the radar volume and the 2DVD site was about $400 \mathrm{~m}$. The radar measurements had a lower time resolution (about $7 \mathrm{~min}$ ) than the 2DVD ( $1 \mathrm{~min}$ ). Additional uncertainty in the comparison was led by different sampling volumes of these two different instruments as well as the evolution of hydrometeor microphysical properties while falling from the radar volume to the 2DVD site. Although sometimes no radar measurement was available while the 2DVD observed rain, overall the radar measurements corresponded well with the synthetic radar measurements calculated from the 2DVD-observed DSDs. Although the radar data were quality controlled, there were some negative $Z_{\mathrm{DR}}$ and $K_{\mathrm{DP}}$ measurements due to random errors and estimation errors, which may have affected the quality of the DSD retrieval.

Figure 11 shows the comparison of the retrieved physical parameters using four different approaches against the observations during the precipitation event on 12 July 2014. The green circles show the EMA-based retrieval using the three-parameter GM, the black triangles show the conventional retrieval using EXP, and the blue squares and the red dots show the results of the EMA-based retrieval using C-G with and without $K_{\mathrm{DP}}$ measurements respectively. The quantitative comparison between radar-derived rain physical parameters and 

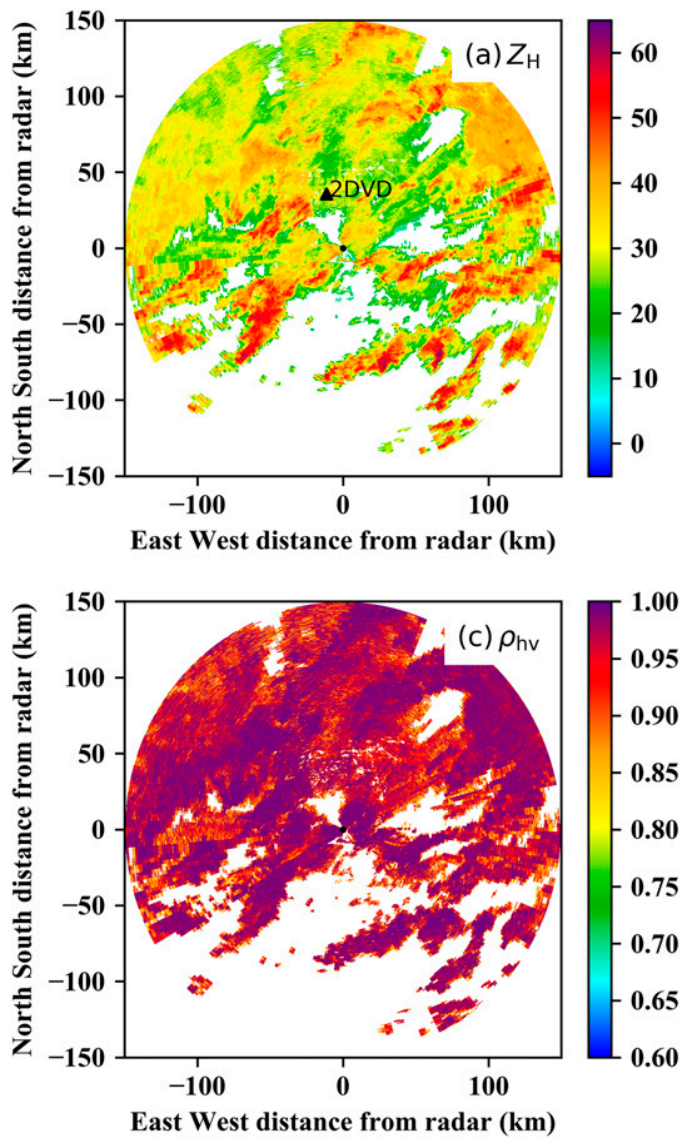

FIG. 9. Plan position indicator (PPI) images at $0.5^{\circ}$ elevation for (a) $Z_{\mathrm{H}}$, (b) $Z_{\mathrm{DR}}$, (c) $\rho_{\mathrm{hv}}$, and (d) $\Phi_{\mathrm{DP}}$ from the Lishui radar at 0640 UTC 12 Jul 2017.

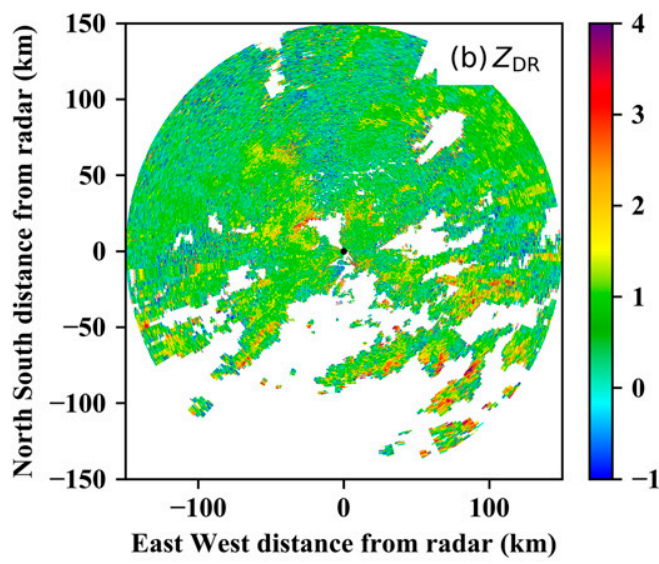

2DVD observations were show in Table 3. For $R$ and $W$ estimates, differences mainly existed when $Z_{\mathrm{H}}$ measurements were large ( $>45 \mathrm{dBZ}$; Fig. 11). When $K_{\mathrm{DP}}$ measurements were not used, the $R$ and $W$ estimates of EMA-based retrieval using C-G showed obvious overestimation, corresponding to the large RMSE and BIAS values. This overestimation also existed in the results of the conventional retrieval using EXP. When the $K_{\mathrm{DP}}$ measurements were used in the retrieval (denoted with blue squares), the results of EMA-based retrieval using C-G were more consistent with the 2DVD observations and the overestimations in $R$ and $W$ were diminished. The results of the above three approaches were not significantly different when the echo intensity was lower $\left(Z_{\mathrm{H}}<45 \mathrm{~dB} Z\right.$; Fig. 11). However, the $R$ and $W$ estimates using three-parameter GM showed larger errors. The corresponding CC values were low, and the errors (FE and RMSE values) were high. In the $D_{m}$ estimates, the results from EMA-based retrieval using C-G with and without $K_{\mathrm{DP}}$ measurements showed comparable accuracy, which was cross verified with the conclusions from section 4 . Even though the $\mathrm{CC}$ values corresponding to

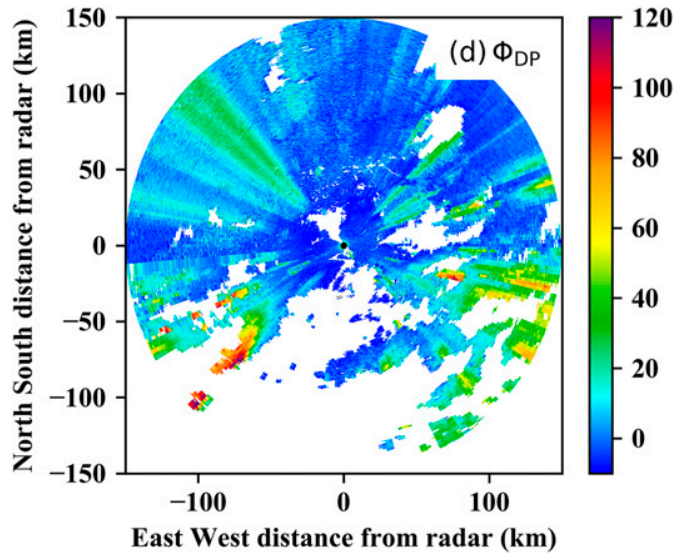

these two kinds of estimates were not high, the low errors (FE, RMSE, and BIAS values) showed generally good consistency with the observation. For the $D_{m}$ values, no valid values were obtained by the conventional retrieval using EXP when $Z_{\mathrm{DR}}$ measurements were negative. In addition, because the constraint in EXP $(\mu=0)$ was not completely suitable for the geographical region in this research, the $D_{m}$ estimates from EXP showed lower accuracy than those from EMAbased retrieval using C-G. The retrieval using the threeparameter GM showed the lowest $\mathrm{CC}$ values and largest errors (FE, RMSE, and BIAS values). The estimates of $N_{t}$ were the worst among the four physical parameters. This is mainly because $N_{t}$ is the zeroth moment of DSDs, which is quite different from the moment orders of radar reflectivity ( $\sim$ sixth moment; Brandes et al. 2004a). This result is also consistent with the results shown in Figs. 4 and 6. The $N_{t}$ estimates from the EMA-based retrieval using $\mathrm{C}-\mathrm{G}$ with and without $K_{\mathrm{DP}}$ measurements showed comparable accuracy, while EXP-based retrieval showed lower accuracy. Then the $N_{t}$ estimates from EMA-based retrieval using the three-parameter 


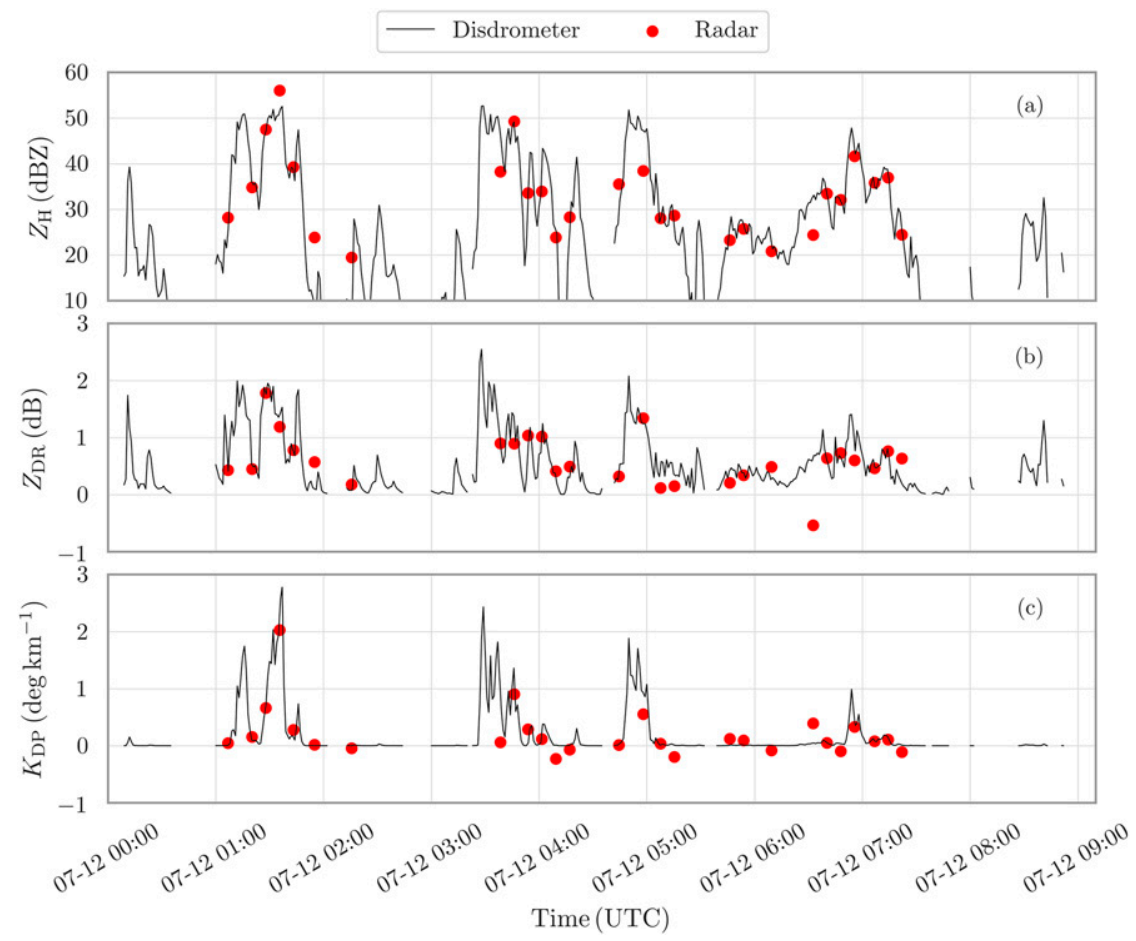

FIG. 10. Comparisons of radar measurements (a) $Z_{\mathrm{H}}$, (b) $Z_{\mathrm{DR}}$, and (c) $K_{\mathrm{DP}}$ denoted with red dots vs those calculated from the 2DVD data (black lines).

GM showed nearly no correlation with the observations $(\mathrm{CC} \sim 0)$. The errors (FE, RMSE, and BIAS values) were also very large when compared with the results of the other methods.

The statistical comparison for another precipitation event that happened on 3-4 July 2014 was also shown in Table 3. In this event, the performance of radar-derived estimates (at $0.5^{\circ}$ elevation) from the four approaches was similar to that in the event on 12 July 2014. The estimates from the EMA-based retrieval using the three-parameter GM also showed very large differences from the observations, which confirmed the conclusions from the simulation experiments. The conventional retrieval using EXP showed much better performance than the three-parameter GM especially for $R, W$, and $D_{m}$. For the EMA-based retrieval using C-G, slight improvements in $R$ and $W$ (decrease in The RMSE and BIAS) were found if the $K_{\mathrm{DP}}$ measurements were included, which was also consistent with the results shown in Fig. 7.

\section{Conclusions and discussion}

Estimating DSD parameters from polarimetric radar data facilitates precipitation microphysics research. It is important to know the error characteristics of the retrieval when using different methods. The three-parameter GM, C-G, and EXP are the three commonly used models for representing rain DSD retrieval. In this study, a general EMA-based approach was used to estimate DSDs based on these models. The performance of the DSD retrievals was investigated through simulation experiments and with real radar data.

First, the feasibility of directly retrieving the DSDs from polarimetric measurements based on the threeparameter GM was studied. The accuracy of the DSD model was determined by comparing the physical parameters $\left(R, W, D_{m}\right.$, and $\left.N_{t}\right)$ estimated from polarimetric data with those calculated from the 2DVD observations. The results based on the C-G and EXP were used as references. It was found that the threeparameter GM was not suitable for directly retrieving DSDs from $Z_{\mathrm{H}}, Z_{\mathrm{DR}}$, and $K_{\mathrm{DP}}$. Even if there was no error in the polarimetric data, the estimates still deviated from the truth because the three-parameter GM does not contain the statistical microphysical characteristics of the DSDs. When there were errors in the radar measurements, the retrievals based on the three-parameter GM were even worse. This unsatisfactory performance prevents the three-parameter GM from being used directly in the retrieval of DSDs. With some additional constraints (e.g., the $\mu-\Lambda$ relation), statistical DSD properties could be retained. Some other DSD models, for example, the generalized 


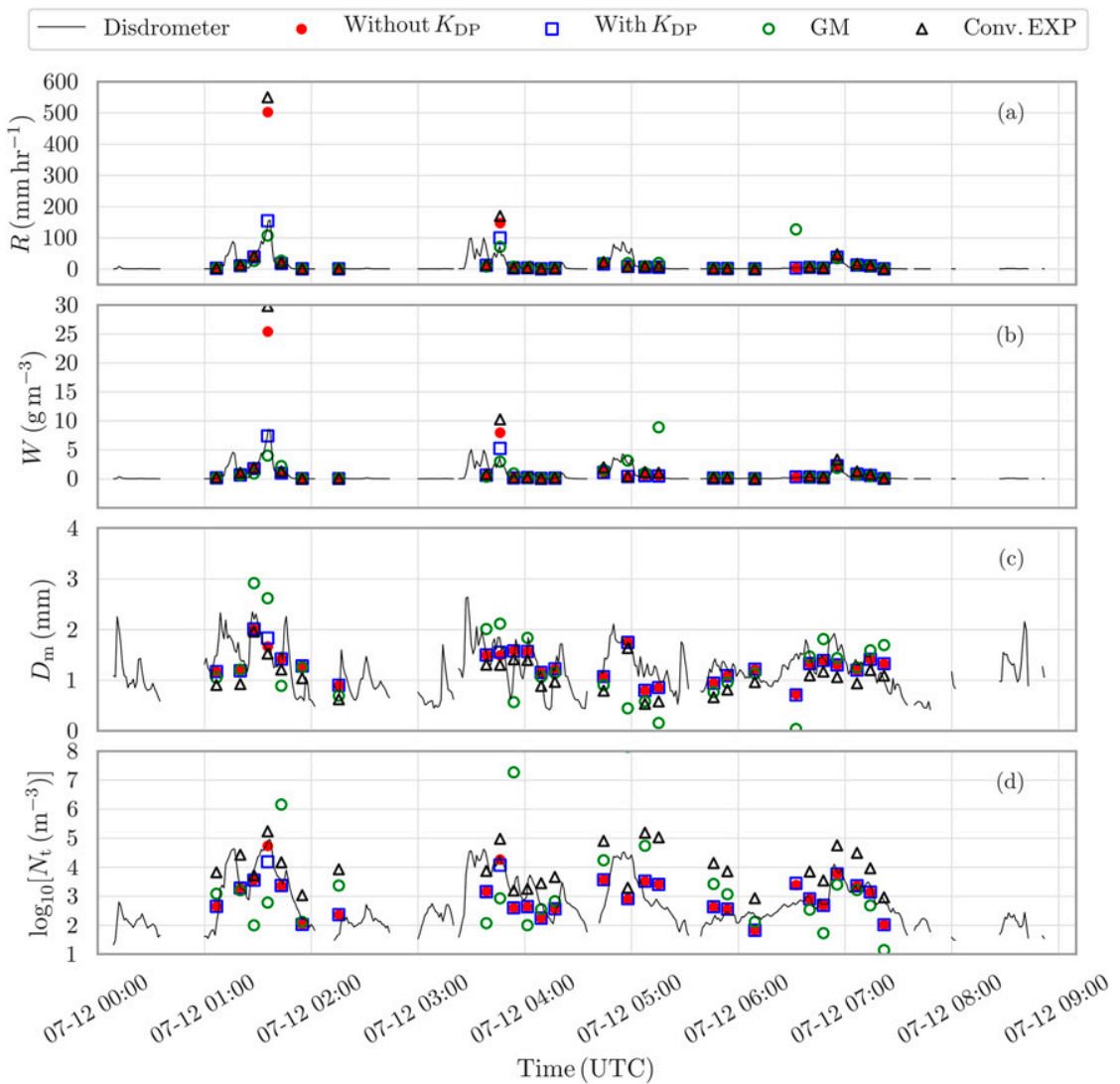

FIG. 11. Comparisons of radar retrieved (a) $R$, (b) $W$, (c) $D_{m}$, and (d) $N_{t}$ with those calculated from the 2DVD data (black lines). The red dots and blue squares represent the results from EMA-based retrieval using C-G with/without $K_{\mathrm{DP}}$ measurements included. The green circles denote the results of EMA-based retrieval using the three-parameter GM and the black triangles denote the results of conventional retrieval using EXP.

gamma distribution with normalization (Lee et al. 2004; Thurai and Bringi 2018) and the gamma distribution constrained by a statistical $D_{m}-\sigma_{m}$ relation (Williams et al. 2014; Zhang 2015), have similar effect.

From the comparisons of the estimated physical parameters with and without $K_{\mathrm{DP}}$ measurements used in the retrievals, it was found that the retrievals based on the EXP became worse when more information was used. Retrieval methods based on $\mathrm{C}-\mathrm{G}$ could make full use of all the polarimetric measurements since the constraints of $\mathrm{C}-\mathrm{G}$ suppressed the effect of $K_{\mathrm{DP}}$ errors and thus performed better than those only using the $Z_{\mathrm{H}}$ and $Z_{\mathrm{DR}}$ measurements. It was also demonstrated that, when errors were contained in $K_{\mathrm{DP}}$ measurements, the accuracy of $W$ could be further enhanced when an accurate $\sigma_{K_{\mathrm{DP}}}$ was used in the EMA, especially when $Z_{\mathrm{H}}>35 \mathrm{~dB} Z$. The $\sigma_{K_{\mathrm{DP}}}$ term in the EMA indicates the reliability of $K_{\mathrm{DP}}$ measurements. If the $K_{\mathrm{DP}}$ is overly trusted ( $\sigma_{K_{\mathrm{DP}}}$ too small), measurement errors can reduce the accuracy of the estimates. On the other hand, if the $\sigma_{K_{\mathrm{DP}}}$ values used are too large, the useful information in $K_{\mathrm{DP}}$ measurements cannot be efficiently utilized. It is therefore important to use an accurately estimated $\sigma_{K_{\mathrm{DP}}}$ to obtain more accurate DSD estimates. The effects of the errors in $Z_{\mathrm{H}}$ and $Z_{\mathrm{DR}}$ measurements were not investigated but are expected to have similar effects.

Finally, polarimetric radar measurements and 2DVD observations from two precipitation events were used to verify the performance of these approaches. The retrieval using the three-parameter GM showed the lowest accuracy as compared with the other three approaches. When only $Z_{\mathrm{H}}$ and $Z_{\mathrm{DR}}$ were used in the EMA-based retrieval using C-G, $R$ and $W$ were sometimes substantially overestimated. However, the overestimation problem was solved by using all three measurements $\left(Z_{\mathrm{H}}, Z_{\mathrm{DR}}\right.$, and $\left.K_{\mathrm{DP}}\right)$ in retrievals, indicating the advantage of making full use of all available information. The conventional retrieval using EXP showed decreased 
TABLE 3. The statistical comparisons of the radar-derived physical parameters $\left(R, W, D_{m}\right.$, and $\left.N_{t}\right)$ and the 2DVD observations in two real events. Four methods were included for comparison (i.e., the EMA-based retrieval using C-G with and without the $K_{\mathrm{DP}}$ included, the EMA-based retrieval using the three-parameter GM, and the conventional retrieval using EXP).

\begin{tabular}{|c|c|c|c|c|c|c|c|c|c|}
\hline & & \multicolumn{4}{|c|}{ 0000-0900 UTC 12 Jul 2014} & \multicolumn{4}{|c|}{1500 UTC 3 Jul-0600 UTC 5 Jul 2014} \\
\hline & & $\begin{array}{c}\text { C-G with } \\
K_{\mathrm{DP}}\end{array}$ & $\begin{array}{c}\text { C-G without } \\
K_{\mathrm{DP}}\end{array}$ & GM & $\begin{array}{c}\text { Conventional } \\
\text { EXP }\end{array}$ & $\begin{array}{c}\text { C-G with } \\
K_{\mathrm{DP}}\end{array}$ & $\begin{array}{c}\text { C-G without } \\
K_{\mathrm{DP}}\end{array}$ & GM & $\begin{array}{c}\text { Conventional } \\
\text { EXP }\end{array}$ \\
\hline \multirow[t]{4}{*}{$R\left(\mathrm{~mm} \mathrm{~h}^{-1}\right)$} & $\mathrm{CC}$ & 0.87 & 0.86 & 0.58 & 0.86 & 0.82 & 0.81 & 0.16 & 0.81 \\
\hline & $\mathrm{FE}$ & 1.25 & 1.35 & 2.68 & 1.97 & 1.05 & 1.06 & 10.84 & 1.41 \\
\hline & RMSE & 16.31 & 64.92 & 28.1 & 74.96 & 5.52 & 7.77 & 25.81 & 9.72 \\
\hline & BIAS & -1.53 & 10.92 & 0.68 & 15.12 & 0.82 & 1.15 & 7.28 & 2.19 \\
\hline \multirow[t]{4}{*}{$W\left(\mathrm{~g} \mathrm{~m}^{-3}\right)$} & $\mathrm{CC}$ & 0.85 & 0.87 & -0.06 & 0.87 & 0.78 & 0.78 & -0.07 & 0.72 \\
\hline & $\mathrm{FE}$ & 1.34 & 1.43 & 65.85 & 2.91 & 1.12 & 1.13 & 408.43 & 2.14 \\
\hline & RMSE & 0.91 & 3.2 & 73.08 & 4.14 & 0.31 & 0.42 & 81.61 & 0.7 \\
\hline & BIAS & -0.06 & 0.59 & 13.08 & 1.05 & 0.06 & 0.08 & 19.17 & 0.23 \\
\hline \multirow[t]{4}{*}{$D_{m}(\mathrm{~mm})$} & $\mathrm{CC}$ & 0.59 & 0.59 & 0.48 & 0.66 & 0.51 & 0.51 & 0.33 & 0.49 \\
\hline & $\mathrm{FE}$ & 0.22 & 0.22 & 0.34 & 0.29 & 0.23 & 0.23 & 0.37 & 0.24 \\
\hline & RMSE & 0.32 & 0.32 & 0.58 & 0.42 & 0.3 & 0.3 & 0.56 & 0.37 \\
\hline & BIAS & -0.09 & -0.1 & -0.15 & -0.31 & 0.01 & 0.01 & -0.14 & -0.19 \\
\hline \multirow[t]{4}{*}{$\log _{10}\left[N_{t}\right]$} & $\mathrm{CC}$ & 0.54 & 0.58 & 0.04 & 0.23 & 0.53 & 0.53 & 0.02 & 0.34 \\
\hline & $\mathrm{FE}$ & 0.18 & 0.17 & 0.54 & 0.41 & 0.18 & 0.18 & 0.72 & 0.38 \\
\hline & RMSE & 7 & 6.86 & 23.91 & 13.14 & 5.71 & 5.72 & 30.19 & 11.79 \\
\hline & BIAS & -1.35 & -1.13 & 6.41 & 9.12 & -1.11 & -1.1 & 11.67 & 8.63 \\
\hline
\end{tabular}

performance because that the model was not tuned for the specific research region and that the measured information was not fully utilized.

The performance of the DSD models depends on what physical parameters we are interested in. For example, besides $W, R, D_{m}$, and $N_{t}$, reflectivity $(Z$, sixth moment of DSD) was also calculated from the DSDs to evaluate the performance of different models. No matter which model was used, all the retrieved $Z$ values were generally close to the real values in all the experiments in sections 2 and 3 . This is mainly because the $Z_{\mathrm{H}}$ measurements are close to the sixth moment of DSD. The retrieval of $Z$ suffered less from the truncation errors and the measurement errors, and the impact of the DSD models was minor. Thus, when evaluating the other DSD models for retrieval, further experiments should be done through comparisons on the physical parameters of interest.

In the above analysis, the gamma distribution constrained by a statistical $\mu-\Lambda$ relation (C-G) had advantages over the unconstrained-gamma distribution in DSD retrieval. However, the C-G also had its own disadvantage because the determination of an accurate statistical $\mu-\Lambda$ relation is not easy. The $\mu$ and $\Lambda$ are not DSD moments and are usually determined by the moment-fitting method (Vivekanandan et al. 2004). Since the DSD moments suffers from the sampling errors of 2DVD (Tokay et al. 2001, 2013, 2014; Thurai et al. 2017), the values of $\mu$ and $\Lambda$ can be biased. To reduce the effect of sampling errors, the moments of median orders were used in estimation of the DSD parameters (the moment-fitting method; Cao and Zhang 2009). The method of sorting and averaging based on two parameters (SATP) might also be a way to mitigate the sampling errors (Cao et al. 2008). Since the sampling errors cannot be completely eliminated by this method, the only way is to minimize its effect as much as possible. In addition, due to the limits of $\Lambda$ (less than 20) used in the EMA analysis, there was a lower limit for $D_{m}$ (about $0.7 \mathrm{~mm}$ ) for the retrieval based on the C-G. In the DSD dataset (21 739 samples) used for simulation, there were 3088 DSD samples (about 14\%) with $D_{m}$ values less than $0.7 \mathrm{~mm}$. Within these samples, only 967 (about 4\%) samples showed larger differences $(>0.2 \mathrm{~mm})$ from the $D_{m}$ estimated based on the C-G model in the simulation experiment with no measurement errors assumed (Fig. 2). Thus, the lower limit due to the C-G model was acceptable considering the other advantages of the C-G.

In addition, explicitly constraining the DSD models by fixed relations is not the only way to introduce physical constraints to retrievals. Based on the Bayesian theorem, the climatic information of the DSD parameters $\left(N_{0}\right.$ and $\left.\Lambda\right)$ was used to obtain better DSD estimates by Cao et al. (2010). Similarly, climatic statistics of $N_{0}, \mu$, and $\Lambda$ can be added to the EMA as a priori information to constrain the retrieval, in which the EMA becomes the variational analysis, which is a potential way to obtain accurate DSD estimates on the basis of the threeparameter GM. In addition, an extra term, which adjusts the DSD parameters to a statistical constraint (e.g., the $\mu-\Lambda$ relation), may be included in the cost function of EMA or a variational analysis. On the other hand, it is 
not necessary to calculate $K_{\mathrm{DP}}$ from $\Phi_{\mathrm{DP}}$ measurements before the DSD retrieval. The $\Phi_{\mathrm{DP}}$ measurements can be directly used if the DSDs in a ray are simultaneously retrieved based on the EMA or a variational analysis.

In the future, a promising way to retrieve DSDs could be to use all the available information, including climatology statistics, all measurements, time evolution of the weather systems, spatiotemporal continuities of precipitation properties, and physical constraints in a statistics-based framework (e.g., EMA, variational analysis) or the Bayesian theorem-based analysis. With the correct characterization of the uncertainties of these sources of information, the DSD parameters and the corresponding physical parameters can be optimized.

Acknowledgments. The authors thank the scientists and engineers working on the 2DVD, Lishui radar, and the other instruments. The authors also thank Dr. Yinghui Lu (PSU) for his careful proofreading of this manuscript. This work was supported by the National Key Research and Development Program of China (Grants 2017YFC1501703 and 2018YFC1506404), the National Natural Science Foundation of China (Grants 41475015, 41875053, and 41675023), the Open Research Program of the State Key Laboratory of Severe Weather, and the Key Research Development Program of Jiangsu Science and Technology Department (Social Development Program, BE2016732).

\section{APPENDIX}

\section{The EMA-Based DSD Retrieval}

Measurements of polarimetric variables can be outside the variables' physical bounds, due to measurement errors. For these measurements, no valid estimates can be obtained using the explicit equation-solving approaches. Extra considerations are required for this kind of problem. The algorithms based on forward operators, which calculate polarimetric variables from DSDs, are suitable for dealing with measurement errors (Rodgers 2000); see Eqs. (1)-(3). Physical constraints are implied in the forward operators, and they help to eliminate the effects of erroneous measurements. Bayesian theory and its variant variational analysis are usually used together with the forward operators to optimally estimate DSDs. Because we did not consider the a priori information in our retrieval, a variant of the variational analysis method was used as a general method for DSD retrieval in this study. In this method, any one of the three-parameter GM, EXP, and C-G distributions can be used as the DSD model. The basic concept can be interpreted as follows. The a priori term in the variational analysis is omitted in the variant and we refer to it as the EMA approach.

First assume a DSD model, the three-parameter GM, EXP, or C-G. Then the DSD parameters are denoted with a state vector $\mathbf{x}, \mathbf{x}=\left[N_{0}, \Lambda\right]^{\mathrm{T}}$ for EXP and C-G and $\mathbf{x}=\left[N_{0}, \Lambda, \mu\right]^{\mathrm{T}}$ for the three-parameter GM. The superscript $\mathrm{T}$ means a transpose. The state vector $\mathbf{x}$ is optimized when the cost function is minimized:

$$
J(\mathbf{x})=\frac{1}{2}[H(\mathbf{x})-\mathbf{y}]^{\mathrm{T}} \mathbf{R}^{-1}[H(\mathbf{x})-\mathbf{y}],
$$

where the forward operators are represented by $H(\cdot)$, which predicts the polarimetric variables $Z_{\mathrm{H}}, Z_{\mathrm{DR}}$, and $K_{\mathrm{DP}}$ from $\mathbf{x} ; \mathbf{y}$ is the observation vector $\left[Z_{\mathrm{H}}, Z_{\mathrm{DR}}\right.$, or $\left.K_{\mathrm{DP}}\right]^{\mathrm{T}}$; and $\mathbf{R}$ is the error covariance of $\mathbf{y}$. In this study, we assumed the errors in $Z_{\mathrm{H}}, Z_{\mathrm{DR}}$, and $K_{\mathrm{DP}}$ were independent and $\mathbf{R}$ was diagonal (Cao et al. 2010), which was expressed as

$$
\mathbf{R}=\left[\begin{array}{ccc}
\sigma_{Z_{\mathrm{H}}}^{2} & 0 & 0 \\
0 & \sigma_{Z_{\mathrm{DR}}}^{2} & 0 \\
0 & 0 & \sigma_{K_{\mathrm{DP}}}^{2}
\end{array}\right],
$$

where $\sigma_{Z_{\mathrm{H}}}, \sigma_{Z_{\mathrm{DR}}}$, and $\sigma_{K_{\mathrm{DP}}}$ are the standard deviations of the errors in $Z_{\mathrm{H}}, Z_{\mathrm{DR}}$, and $K_{\mathrm{DP}}$. The forward model $H(\cdot)$ first calculates $N(D)$ from $\mathbf{x}$ based on the assumed DSD model, and then calculates $Z_{\mathrm{H}}, Z_{\mathrm{DR}}$, and $K_{\mathrm{DP}}$ using Eqs. (1)-(3). To minimize Eq. (A1), its corresponding gradient is given by

$$
\mathbf{g}(\mathbf{x})=\mathbf{H}^{\mathrm{T}} \mathbf{R}^{-1}[H(\mathbf{x})-\mathbf{y}],
$$

where $\mathbf{H}$ is the Jacobian matrix composed of the partial derivative of the predicted variables. As we know, the forward operators are nonlinear, so $\mathbf{x}$ is minimized iteratively using the linearized forward operators $H(\mathbf{x})=H\left(\mathbf{x}_{k}\right)+H^{\mathrm{T}} \times\left(\mathbf{x}-\mathbf{x}_{k}\right)$, where the subscript of $\mathbf{x}$ means $\mathbf{x}$ at the $k$ th iteration. By setting the gradient to zero, the optimized $\mathbf{x}$, which minimizes the linearized cost function at the $k+$ first iteration, is given by

$$
\mathbf{x}_{k+1}=\mathbf{x}_{k}+\mathbf{A}^{-1}\left(\mathbf{H}^{\mathrm{T}} \mathbf{R}^{-1} \delta \mathbf{y}\right),
$$

where $=\mathbf{H}^{\mathrm{T}} \mathbf{R}^{-1} \mathbf{H}$, and $\delta \mathbf{y}=\mathbf{y}-H(\mathbf{x})$. We use $N_{0}=$ $8 \times 10^{3} \mathrm{~m}^{-3} \mathrm{~mm}^{-1}, \Lambda=5 \mathrm{~mm}^{-1}$, and $\mu=0$ for the first iteration. Note that the optimization method is not very sensitive to the choice of these values. A $\chi^{2}$ convergence test can be used for the termination of the iteration. In this study, we used another way to solve the problem, which was to minimize Eq. (A1) directly using the bounded nonlinear optimization algorithm, for example, the limited-memory Broyden-Fletcher-GoldfarbShanno algorithm for bound-constrained large-scale 
nonlinear optimization (L-BFGS-B; Byrd et al. 1995). With Eqs. (A1) and (A3), the Hessian is approximately estimated with the optimization procedures. One of the advantages of these bounded optimization algorithms is their ability to constrain the DSD parameter solutions within given upper and lower limits to avoid solutions without a physical meaning. The limits for the DSD parameters used in this study were $10^{-4} \leq N_{0} \leq 10^{15}$, $0.01 \leq \Lambda \leq 20$, and $-3.5 \leq \mu \leq 15$ (Cao et al. 2008). The DSD parameters and $N(D)$ were considered to be statistically optimized when the state variable $\mathbf{x}$ minimized Eq. (A1) (Rodgers 2000). Note that, for C-G and EXP, $Z_{\mathrm{DR}}$ monotonically changes with $\Lambda ; Z_{\mathrm{H}}$ and $K_{\mathrm{DP}}$ monotonically change with $\Lambda\left(N_{0}\right)$ if $N_{0}(\Lambda)$ is fixed. Thus, there is a one-to-one mapping between the DSD parameter sets and the polarimetric variables. In such a case, the cost function is concave and EMA analysis can always reach global minimization. For the threeparameter GM, similar monotonic relationships between the polarimetric variables and DSD parameters generally exist except when $K_{\mathrm{DP}}\left(Z_{\mathrm{DR}}\right)$ is close to $0^{\circ} \mathrm{km}^{-1}(0 \mathrm{~dB})$. When $K_{\mathrm{DP}}\left(Z_{\mathrm{DR}}\right)$ is close to $0^{\circ} \mathrm{km}^{-1}(0 \mathrm{~dB})$, nonmonotonicity can exist. Thus, the cost function is linearized and converted to a quadratic function in this study, which helps to avoid the local minimization.

One of the keys for EMA-based retrieval is its error terms $\left(\sigma_{Z_{\mathrm{H}}}, \sigma_{Z_{\mathrm{DR}}}\right.$, and $\left.\sigma_{K_{\mathrm{DP}}}\right)$. They denote the reliability of the measurements. If we assume no errors exist in the polarimetric data, the corresponding error terms should be very small, for example, $\sigma_{Z_{\mathrm{H}}}=0.0001 \mathrm{~dB}, \sigma_{Z_{\mathrm{DR}}}=$ $0.00002 \mathrm{~dB}$, and $\sigma_{K_{\mathrm{DP}}}=0.00003^{\circ} \mathrm{km}^{-1}$. For practical applications, the error terms denote the measurement errors, which are mainly decided from the radar digital signal processing or estimation processes. If one of the error terms is enlarged, the corresponding polarimetric measurements are less trusted and less information is contained in the results.

\section{REFERENCES}

Anagnostou, M. N., J. Kalogiros, F. S. Marzano, E. N. Anagnostou, M. Montopoli, and E. Piccioti, 2013: Performance evaluation of a new dual-polarization microphysical algorithm based on longterm X-band radar and disdrometer observations. J. Hydrometeor., 14, 560-576, https://doi.org/10.1175/JHM-D-12-057.1.

Brandes, E. A., G. Zhang, and J. Vivekanandan, 2002: Experiments in rainfall estimation with a polarimetric radar in a subtropical environment. J. Appl. Meteor., 41, 674-685, https://doi.org/ 10.1175/1520-0450(2002)041<0674:EIREWA > 2.0.CO;2.

- —, and — 2004a: Drop size distribution retrieval with polarimetric radar: Model and application. J. Appl. Meteor., 43, 461-475, https://doi.org/10.1175/1520-0450(2004)043<0461: DSDRWP $>2.0 . \mathrm{CO} ; 2$.

,$- \ldots$, and $\longrightarrow, 2004 \mathrm{~b}$ : Comparison of polarimetric radar drop size distribution retrieval algorithms. J. Atmos. Oceanic
Technol., 21, 584-598, https://doi.org/10.1175/1520-0426(2004) $021<0584$ :COPRDS $>2.0 . \mathrm{CO} ; 2$.

Bringi, V. N., and V. Chandrasekar, 2001: Polarimetric Doppler Weather Radar: Principles and Applications. Cambridge University Press, 664 pp.

— , G.-J. Huang, V. Chandrasekar, and E. Gorgucci, 2002: A methodology for estimating the parameters of a gamma raindrop size distribution model from polarimetric radar data: Application to a squall-line event from the TRMM/Brazil campaign. J. Atmos. Oceanic Technol., 19, 633-645, https:// doi.org/10.1175/1520-0426(2002)019<0633:AMFETP>2.0.CO;2. , L. Tolstoy, M. Thurai, and W. A. Petersen, 2015: Estimation of spatial correlation of drop size distribution parameters and rain rate using NASA's S-band polarimetric radar and 2D video disdrometer network: Two case studies from MC3E. J. Hydrometeor., 16, 1207-1221, https://doi.org/10.1175/JHM-D-14-0204.1.

Byrd, R. H., P. Lu, J. Nocedal, and C. Zhu, 1995: A limited memory algorithm for bound constrained optimization. SIAM J. Sci. Comput., 16, 1190-1208, https://doi.org/10.1137/0916069.

Cao, Q., and G. Zhang, 2009: Errors in estimating raindrop size distribution parameters employing disdrometer and simulated raindrop spectra. J. Appl. Meteor. Climatol., 48, 406-425, https://doi.org/10.1175/2008JAMC2026.1.

$\longrightarrow,-$, E. Brandes, T. Schuur, A. Ryzhkov, and K. Ikeda, 2008: Analysis of video disdrometer and polarimetric radar data to characterize rain microphysics in Oklahoma. J. Appl. Meteor. Climatol., 47, 2238-2255, https://oi.org/10.1175/2008JAMC1732.1.

,,--- , and -2010 : Polarimetric radar rain estimation through retrieval of drop size distribution using a Bayesian approach. J. Appl. Meteor. Climatol., 49, 973-990, https:// doi.org/10.1175/2009JAMC2227.1.

_ - M. B. Yeary, and G. Zhang, 2012: Efficient ways to learn weather radar polarimetry. IEEE Trans. Educ., 55, 58-68, https://doi.org/10.1109/TE.2011.2118211.

Doviak, R. J., and D. S. Zrnić, 1993: Doppler Radar and Weather Observations. 2nd ed. Academic Press, 562 pp.

Giangrande, S. E., R. McGraw, and L. Lei, 2013: An application of linear programming to polarimetric radar differential phase processing. J. Atmos. Oceanic Technol., 30, 1716-1729, https:// doi.org/10.1175/JTECH-D-12-00147.1.

Gorgucci, E., V. Chandrasekar, V. N. Bringi, and G. Scarchilli, 2002: Estimation of raindrop size distribution parameters from polarimetric radar measurements. J. Atmos. Sci., 59, 2373-2384, https:// doi.org/10.1175/1520-0469(2002)059<2373:EORSDP>2.0.CO;2.

Gosset, M., E.-P. Zahiri, and S. Moumouni, 2010: Rain drop size distribution variability and impact on X-band polarimetric radar retrieval: Results from the AMMA campaign in Benin. Quart. J. Roy. Meteor. Soc., 136, 243-256, https://doi.org/ 10.1002/qj.556.

Huang, H., G. Zhang, K. Zhao, and S. E. Giangrande, 2017: A hybrid method to estimate specific differential phase and rainfall with linear programming and physics constraints. IEEE Trans. Geosci. Remote Sens., 55, 96-111, https://doi.org/10.1109/TGRS.2016.2596295.

— , and Coauthors, 2018: Quantitative precipitation estimation with operational polarimetric radar measurements in southern China: A differential phase-based variational approach. J. Atmos. Oceanic Technol., 35, 1253-1271, https://doi.org/ 10.1175/JTECH-D-17-0142.1.

Kalogiros, J., M. N. Anagnostou, E. N. Anagnostou, M. Montopoli, E. Picciotti, and F. S. Marzano, 2013: Optimum estimation of rain microphysical parameters from X-band dual-polarization radar observables. IEEE Trans. Geosci. Remote Sens., 51, 3063-3076, https://doi.org/10.1109/TGRS.2012.2211606. 
Kumjian, M. R., and A. V. Ryzhkov, 2010: The impact of evaporation on polarimetric characteristics of rain: Theoretical model and practical implications. J. Appl. Meteor. Climatol., 49, 1247-1267, https://doi.org/10.1175/2010JAMC2243.1.

_ , and O. P. Prat, 2014: The impact of raindrop collisional processes on the polarimetric radar variables. J. Atmos. Sci., 71, 3052-3067, https://doi.org/10.1175/JAS-D-13-0357.1.

Lee, G. W., 2006: Sources of errors in rainfall measurements by polarimetric radar: Variability of drop size distributions, observational noise, and variation of relationships between $R$ and polarimetric parameters. J. Atmos. Oceanic Technol., 23, 1005-1028, https://doi.org/10.1175/JTECH1899.1.

I. Zawadzki, W. Szyrmer, D. Sempere-Torres, and R. Uijlenhoet, 2004: A general approach to double-moment normalization of drop size distributions. J. Appl. Meteor., 43, 264-281, https://doi.org/10.1175/1520-0450(2004)043<0264: AGATDN $>2.0 . \mathrm{CO} ; 2$.

Maesaka, T., K. Iwanami, and M. Maki, 2012: Non-negative $K_{\mathrm{DP}}$ estimation by monotone increasing $\Phi_{\mathrm{DP}}$ assumption below melting layer. Preprints, Seventh European Conf. on Radar in Meteorology and Hydrology, Toulouse, France, Météo France, 26 QPE.

Mishchenko, M. I., L. D. Travis, and D. W. Mackowski, 1996: $T$-matrix computations of light scattering by nonspherical particles: A review. J. Quant. Spectrosc. Radiat. Transfer, $\mathbf{5 5}$, 535-575, https://doi.org/10.1016/0022-4073(96)00002-7.

Moisseev, D. N., and V. Chandrasekar, 2007: Examination of the $\mu-\Lambda$ relation suggested for drop size distribution parameters. J. Atmos. Oceanic Technol., 24, 847-855, https://doi.org/10.1175/ JTECH2010.1.

Morrison, H., M. R. Kumjian, C. P. Martinkus, O. P. Prat, and M. van Lier-Walqui, 2019: A general $N$-moment normalization method for deriving rain drop size distribution scaling relationships. J. Appl. Meteor. Climatol., 58, 247-267, https:// doi.org/10.1175/JAMC-D-18-0060.1.

Park, S. G., M. Maki, K. Iwanami, V. N. Bringi, and V. Chandrasekar, 2005: Correction of radar reflectivity and differential reflectivity for rain attenuation at X band. Part II: Evaluation and application. J. Atmos. Oceanic Technol., 22, 1633-1655, https://doi.org/ 10.1175/JTECH1804.1.

Raupach, T. H., and A. Berne, 2017: Retrieval of the raindrop size distribution from polarimetric radar data using doublemoment normalisation. Atmos. Meas. Tech., 10, 2573-2594, https://doi.org/10.5194/amt-10-2573-2017.

Rodgers, C. D., 2000: Inverse Methods for Atmospheric Sounding: Theory and Practice. Series on Atmospheric, Oceanic and Planetary Physics, Vol. 2, World Scientific, $238 \mathrm{pp}$.

Ryzhkov, A. V., S. Giangrande, and T. Schuur, 2003: Rainfall measurements with the polarimetric WSR-88D radar. NOAA Rep., 99 pp.

Seliga, T. A., and V. N. Bringi, 1976: Potential use of radar differential reflectivity measurements at orthogonal polarizations for measuring precipitation. J. Appl. Meteor., 15, 69-76, https://doi.org/ 10.1175/1520-0450(1976)015<0069:PUORDR > 2.0.CO;2.

Testud, J., S. Oury, R. A. Black, P. Amayenc, and X. Dou, 2001: The concept of "normalized" distribution to describe raindrop spectra: A tool for cloud physics and cloud remote sensing. J. Appl. Meteor., 40, 1118-1140, https://doi.org/ 10.1175/1520-0450(2001)040<1118:TCONDT > 2.0.CO;2.

Thurai, M., and V. N. Bringi, 2018: Application of the generalized gamma model to represent the full rain drop size distribution spectra. J. Appl. Meteor. Climatol., 57, 1197-1210, https:// doi.org/10.1175/jamc-d-17-0235.1.
, P. Gatlin, V. N. Bringi, W. Petersen, P. Kennedy, B. Notaros, and L. Carey, 2017: Toward completing the raindrop size spectrum: Case studies involving $2 \mathrm{D}$-video disdrometer, droplet spectrometer, and polarimetric radar measurements. J. Appl. Meteor. Climatol., 56, 877-896, https:// doi.org/10.1175/JAMC-D-16-0304.1.

Tokay, A., A. Kruger, and W. F. Krajewski, 2001: Comparison of drop size distribution measurements by impact and optical disdrometers. J. Appl. Meteor., 40, 2083-2097, https://doi.org/ 10.1175/1520-0450(2001)040<2083:CODSDM >2.0.CO;2.

, W. A. Petersen, P. Gatlin, and M. Wingo, 2013: Comparison of raindrop size distribution measurements by collocated disdrometers. J. Atmos. Oceanic Technol., 30, 1672-1690, https://doi.org/10.1175/JTECH-D-12-00163.1.

— D. B. Wolff, and W. A. Petersen, 2014: Evaluation of the new version of the laser-optical disdrometer, OTT Parsivel ${ }^{2}$. J. Atmos. Oceanic Technol., 31, 1276-1288, https://doi.org/ 10.1175/JTECH-D-13-00174.1.

Ulbrich, C. W., 1983: Natural variations in the analytical form of the raindrop size distribution. J. Climate Appl. Meteor., 22, 1764-1775, https://doi.org/10.1175/1520-0450(1983)022<1764: NVITAF $>2.0 . C O ; 2$.

Vivekanandan, J., W. M. Adams, and V. N. Bringi, 1991: Rigorous approach to polarimetric radar modeling of hydrometeor orientation distributions. J. Appl. Meteor., 30, 1053-1063, https:// doi.org/10.1175/1520-0450(1991)030<1053:RATPRM >2.0.CO;2. , G. Zhang, S. M. Ellis, D. Rajopadhyaya, and S. K. Avery, 2003: Radar reflectivity calibration using differential propagation phase measurement. Radio Sci., 38, 14, https://doi.org/ 10.1029/2002RS002676.

—_ - _ and E. Brandes, 2004: Polarimetric radar estimators based on a constrained gamma drop size distribution model. J. Appl. Meteor., 43, 217-230, https://doi.org/ 10.1175/1520-0450(2004)043<0217:PREBOA > 2.0.CO;2.

Wen, L., K. Zhao, G. Zhang, M. Xue, B. Zhou, S. Liu, and X. Chen, 2016: Statistical characteristics of raindrop size distributions observed in East China during the Asian summer monsoon season using 2-D video disdrometer and Micro Rain Radar data. J. Geophys. Res. Atmos., 121, 2265-2282, https://doi.org/ 10.1002/2015JD024160.

Williams, C. R., and Coauthors, 2014: Describing the shape of raindrop size distributions using uncorrelated raindrop mass spectrum parameters. J. Appl. Meteor. Climatol., 53, 12821296, https://doi.org/10.1175/JAMC-D-13-076.1.

Zhang, G., 2015: Comments on "Describing the shape of raindrop size distributions using uncorrelated raindrop mass spectrum parameters." J. Appl. Meteor. Climatol., 54,1970-1976, https:// doi.org/10.1175/JAMC-D-14-0210.1.

2016: Weather Radar Polarimetry. 1st ed. CRC Press, 322 pp.

_ J. Vivekanandan, and E. Brandes, 2001: A method for estimating rain rate and drop size distribution from polarimetric radar measurements. IEEE Trans. Geosci. Remote Sens., 39, 830-841, https://doi.org/10.1109/36.917906.

$,-\longrightarrow,-$, R. Meneghini, and T. Kozu, 2003: The shapeslope relation in observed gamma raindrop size distributions: Statistical error or useful information? J. Atmos. Oceanic Technol., 20, 1106-1119, https://doi.org/10.1175/1520-0426(2003) $020<1106$ :TSRIOG $>2.0$. CO 2 .

, J. Sun, and E. A. Brandes, 2006: Improving parameterization of rain microphysics with disdrometer and radar observations. J. Atmos. Sci., 63, 1273-1290, https://doi.org/10.1175/JAS3680.1. 